\title{
New perspective on the $U(n)$ Wigner-Racah calculus: II. Elementary reduced Wigner coefficients for $U(n)$
}

\author{
R Le Blanc $\dagger$ and $\mathrm{K} T$ Hecht $\ddagger$ \\ Department of Physics, University of Michigan, Ann Arbor, MI 48109, USA
}

Received 16 September 1986, in final form 11 March 1987

\begin{abstract}
Exploiting the powerful formalism of the vector coherent state theory expounded in part I, the group theoretical content of the complementarity principle and a novel interpretation of the operator pattern of Biedenharn and Louck, we rederive in a simple fashion all $\mathrm{U}(n): \mathrm{U}(n-1)$ reduced Wigner coefficients for the elementary $\mathrm{U}(n)$ tensors.
\end{abstract}

\section{Introduction}

In their successful programme to give a full implementation of the Wigner-Racah calculus for the unitary groups, $\mathrm{U}(n)$, Biedenharn and Louck (see the review article by Louck (1970)) introduced a set of abstract tensor operators which carry the basic structure of this calculus. The recent realisation (Le Blanc and Rowe 1986a, b) that a strict group theoretical meaning can be assigned to the operator pattern used in their classification has led to the possibility of their very explicit construction in an $(n-1) \times$ $n$-dimensional space of Bargmann variables (Bargmann 1962).

We thus make use of this recent development to propose a new approach simplifying to a large extent tensorial computations in Bargmann spaces. We exploit the powerful formalism of the vector coherent state theory expounded in part I (Hecht et al 1987b), the group theoretical content of the complementarity principle and this novel interpretation of the operator pattern of Biedenharn and Louck to rederive in a simple fashion all $\mathrm{U}(n): \mathrm{U}(n-1)$ reduced Wigner coefficients for the elementary $\left\{\dot{1}_{k} \dot{0}\right\}_{n} \mathrm{U}(n)$ tensors, coefficients first derived by Biedenharn and Louck (1968) using arduous boson poly. nomial manipulations.

These coefficients, being the basic building blocks for the construction of generic $\mathrm{U}(n)$ tensors, are fundamental to any attempt to unravel in a canonical way the outer multiplicity problem for the unitary groups. In fact, these coefficients are at the root of the elaborate structure built up by Biedenharn and Louck in order to give a canonical foundation to the $U(n)$ Wigner-Racah calculus. A more straightforward rederivation of their values is thus a necessary first step in any attempt to improve on their results. We recall that theirs is an elaborate structure articulated on what they have coined as the 'pattern calculus'.

\footnotetext{
† NSERC Postdoctoral Fellow, now at A W Wright Nuclear Structure Laboratory, Yale University, New Haven, CT 06520, USA.

$\ddagger$ Supported in part by US National Science Foundation.
} 
We thus confirm but also enlarge on the results of Biedenharn and Louck since we unravel the simple structure of these coefficients. By structure, we not only mean their algebraic expressions but also the nature of their basic components. In fact, we show that the elementary reduced Wigner coefficients are the product of a multiplicityfree $\mathrm{U}(n-1)$ Racah coefficient times some simple dimensional factors and normalisation factors originating from the vector coherent state theory of $I$. We note that the present approach has already been used with similar end results for the computation of the fundamental Wigner coefficients for the non-canonical group chains $\mathrm{SU}(3) \supset \mathrm{O}(3)$ (Le Blanc and Rowe 1985a, b) and $\mathrm{SU}(4) \supset \mathrm{O}(4)$ (Hecht et al 1987a).

\section{Bargmann tensors in a $U(n-1) \times U(n)$ Bargmann space}

The elementary $U(n)$ tensors (Biedenharn and Louck 1968) to be constructed below act in $\mathrm{U}(n)$ bases classified according to the canonical subgroup chain

$$
\mathrm{U}(n) \supset \mathrm{U}(n-1) \supset \ldots \supset \mathrm{U}(1) .
$$

Basis states belonging to a unirrep $\{m\}_{n}=\left\{m_{1 n}, m_{2 n}, \ldots, m_{n n}\right\}$ of $\mathrm{U}(n)$ will be enumerated by a Gel'fand pattern

$$
\left|\begin{array}{c}
\{m\}_{n} \\
\{m\}_{n-} \\
\vdots \\
m_{11}
\end{array}\right\rangle
$$

It will be assumed that the reader is familiar with the Gel'fand notation and the resolution of the weight multiplicity problem in terms of the so-called 'betweenness' conditions on the pattern entries (see, e.g., Louck 1970).

The explicit construction of the elementary $U(n)$ tensors of this investigation leans heavily on the equivalence of $\mathrm{SU}(n)$ and $\mathrm{U}(n)$. It is well known that one needs at most $d$ Bargmann $n$-vectors

$$
\boldsymbol{g}^{i}=\left(g_{1}^{i}, g_{2}^{i}, \ldots, g_{n}^{i}\right) \quad 1 \leqslant i \leqslant d
$$

in order to achieve the construction of a basis of Bargmann states spanning a $d$-rowed unirrep of $\mathrm{U}(n)$, where we refer to $\mathrm{U}(n)$ representations with

$$
m_{d n} \neq 0 \quad m_{d+1, n}, m_{d+2, n}, \ldots, m_{n n}=0
$$

as $d$-rowed representations of $\mathrm{U}(n)$. Generic $\mathrm{SU}(n)$ unirreps, being equivalent to $d=(n-1)$-rowed irreps of $\mathrm{U}(n)$, can thus be built in terms of $n-1 \mathrm{U}(n)$ Bargmann vectors.

Polynomials of degree $k$ in the $g_{j}^{i}$ carry a totally symmetric representation $\{k \dot{0}\}_{d n}$ of the group $\mathrm{U}(d n)$. (As in I, a dot over an index signifies its repetition, and in this case indicates $d n-1$ zeros.) Consideration of the transformation properties of these polynomials under the upper $\mathrm{U}(d)$ and lower $\mathrm{U}(n)$ group actions and the use of the complementarity theorem (Baird and Biedenharn 1963, Moshinsky 1963) show that under the decomposition

$$
\mathrm{U}(d n) \downarrow \mathrm{U}(d) \times \mathrm{U}(n):\{k \dot{0}\}_{d n} \downarrow=\sum_{\{m\}}\{m\}_{d} \times\{m\}_{n} \quad \sum_{i=1}^{d} m_{i n}=k
$$


with

$\{m\}_{d} \times\{m\}_{n}=\left\{m_{1 n} m_{2 n} \ldots m_{d n}\right\}_{d} \times\left\{m_{1 n} m_{2 n} \ldots m_{d n} 0 \ldots 0\right\}_{n}$

the polynomials can be labelled by a double Gel'fand pattern

$$
\left\langle g\left(\begin{array}{c}
m^{\prime} \\
\{m\}_{n} \\
m
\end{array}\right)\right.
$$

In the present application, with $d=n-1$, the states of interest will be those where the upper pattern is restricted to being maximal in its $\mathrm{U}(n-1)$ subrepresentations, i.e. the $\mathrm{U}(n)$ entry and the $\mathrm{U}(n-1)$ upper entry of the double pattern are given by the same partition

$$
\begin{aligned}
& \{m\}_{n-1}^{\text {upper }}=\left\{m_{1 n}, m_{2 n}, \ldots, m_{n-1, n}\right\}^{\text {upper }} \\
& \{m\}_{n}=\left\{m_{1 n}, m_{2 n}, \ldots, m_{n-1, n}, 0\right\} .
\end{aligned}
$$

In a full $\mathrm{U}(n) \times \mathrm{U}(n)$ Bargmann space, the Bargmann polynomial of highest weight under both upper and lower group actions is given by (Baird and Biedenharn 1963, Moshinsky 1963)

$\left\langle\begin{array}{c}\mathrm{hW} \\ g m\}_{n} \\ \mathrm{hW}\end{array}\right\rangle=N^{-1}\left(\{m\}_{n}\right)\left(g_{1}^{1}\right)^{m_{1 n}-m_{2 n}}\left(g_{12}^{12}\right)^{m_{2 n}-m_{3 n}} \ldots\left(g_{12 \ldots n-1}^{12 \ldots n-1}\right)^{m_{n-1, n}-m_{n n}}\left(g_{12 \ldots n}^{12 \ldots n}\right)^{m_{n n}}$

with

$$
g_{12 \ldots k}^{12 \ldots k}=\left|\begin{array}{cccc}
g_{1}^{1} & g_{2}^{1} & \ldots & g_{k}^{1} \\
g_{1}^{2} & g_{2}^{2} & \ldots & g_{k}^{2} \\
\vdots & \vdots & \ddots & \vdots \\
g_{1}^{k} & g_{2}^{k} & \cdots & g_{k}^{k}
\end{array}\right|
$$

Here, $N\left(\{m\}_{n}\right)$ is a normalisation factor given by

$$
N^{2}\left(\{m\}_{n}\right)=\frac{\Pi_{l=1}^{n} p_{l n} !}{\Pi_{k<1}^{n}\left(p_{k n}-p_{l n}\right)}
$$

with $p_{i j}=m_{i j}+j-i$ and where we agree to take the positive square root. It is verified that, with $m_{n n}$ set to zero, the highest weight state (2.6) is also the highest weight state for the group product $\mathrm{U}(n-1) \times \mathrm{U}(n)$.

In order to provide a canonical resolution to the outer multiplicity problem, Biedenharn, Louck and collaborators, in a series of articles (see Louck 1970, Biedenharn and Louck 1972, Louck and Biedenharn 1973, Biedenharn et al 1972, 1985 and references therein) developed an elaborate formalism through which they postulate the existence of an abstract set of $\operatorname{SU}(n)$ tensor operators $\{\mathscr{T}\}$ classified by upper (operator) patterns. This set is denoted

$$
\mathscr{T}\left(\begin{array}{c}
\Gamma \\
\{m\}_{n} \\
m
\end{array}\right)
$$

where $\Gamma$ is an operator (inverted Gel'fand) pattern and where the lower pattern $m$ is a usual lower $U(n-1)$ Gel'fand pattern which labels a basis for the $U(n)$ tensorial 
unirrep $\{m\}_{n}$. The operator pattern is supposed to characterise the structural and tensorial properties of $\mathscr{T}$. The most important property for the purpose of this work is the fact that the upper pattern specifies the shift properties of the tensor, i.e. when applied to a state belonging to the $U(n)$ unirrep $\left\{m^{o}\right\}_{n}$, the tensor $\mathscr{T}$ maps this state to a new unirrep labelled by

$$
\mathscr{T}\left(\begin{array}{c}
\Gamma \\
\{m\}_{n}
\end{array}\right):\left\{m^{\circ}\right\}_{n} \rightarrow\left\{m^{\circ}+\Delta\right\}_{n}
$$

where the shift $\Delta$ is an $n$-vector

$$
\Delta=\left(\Delta_{1}, \Delta_{2}, \ldots, \Delta_{n}\right)
$$

the components of which are given by

$$
\Delta_{j}=\sum_{i=1}^{j} \Gamma_{i j}-\sum_{i=1}^{j-1} \Gamma_{i, j-1} \quad \Delta_{1}=\Gamma_{11}
$$

Other important properties like the null-space property, the inclusion property and the limit properties, relevant to the solution of a generic multiplicity case, are thoroughly reviewed in the above-mentioned references and will be of no immediate concern to us since we will only consider here the coupling of multiplicity-free tensors, more precisely the elementary and fundamental tensors, for which these concepts need not be enlarged upon.

The elementary abstract $U(n)$ tensors are identified by the partition $\left\{\dot{1}_{k} \dot{0}\right\}_{n}, 1 \leqslant k \leqslant$ $n-1$, with associated Young tableaux forming a vertical array of $k$ boxes representing an antisymmetrised tensor of rank $k$. The selection rule for their multiplicity-free coupling is simple: in a Kronecker product the boxes are added to $k$ of the rows of the original tableau specified by the upper pattern, with at most one box added to a given row. The coupling is represented by

$$
\mathscr{T}\left\langle\begin{array}{c}
\Gamma \\
\left\{\dot{1}_{k} \dot{0}\right\}_{n}
\end{array}\right):\left\{m^{o}\right\}_{n} \rightarrow\left\{m^{o}+\Delta^{(k)}\left(i_{1}, i_{2}, \ldots, i_{k}\right)\right\}_{n} .
$$

In $(2.10 a)$

$$
\Delta^{(k)}\left(i_{1}, i_{2}, \ldots, i_{k}\right)
$$

is the $n$-vector given by $(2.9 c)$ and has vanishing entries everywhere except for the $k$ components $\left(i_{1}, i_{2}, \ldots, i_{k}\right)$ which have value unity. The $n$-vector $\Delta^{(k)}\left(i_{1}, i_{2}, \ldots, i_{k}\right)$ is thus a permutation of the $n$-vector $\left(i_{k} \dot{0}\right)$. The fundamental tensors, with $k=1$, are a special case of the elementary tensors, with $\Delta^{(1)}(i)$ specifying the index $i$ of the row to which the single box is added in the Kronecker product. Note that, for the elementary tensors and, more generally, for any multiplicity-free coupling, there is a one-to-one correspondence between the upper pattern and the shift $\Delta$. Therefore, the shift $\Delta^{(k)}\left(i_{1}, i_{2}, \ldots, i_{k}\right)$ will here uniquely identify the parent tensor. To be consistent with the appendix, we adopt the convention

$$
1 \leqslant i_{1}<i_{2}<\ldots<i_{k} \leqslant n
$$

In the above discussion, the elementary shift tensors $\mathscr{T}$ were abstract objects without a specific realisation. Their explicit realisations $T$ in a $\mathrm{U}(n-1) \times \mathrm{U}(n)$ Bargmann space can be given in terms of (multiplicative and/or differential) Bargmann operators. This was proved for $\mathrm{SU}(3)$ (i.e. $n=3$ ) by Le Blanc and Rowe (1986a, b) and the generalisation to $\mathrm{U}(n-1) \times \mathrm{U}(n)$ is straightforward. For example, the $n-1$ vectors $\boldsymbol{g}^{1}, \boldsymbol{g}^{2}, \ldots, \boldsymbol{g}^{n-1}$ can be shown to be shift tensors with respective shifts

$$
\Delta^{(1)}(10 \ldots 0), \Delta^{(1)}(010 \ldots 0), \ldots, \Delta^{(1)}(0 \ldots 010)
$$


but only when acting between $\mathrm{U}(n-1) \times \mathrm{U}(n)$ states of highest weight in their upper patterns. Similarly, the unnormalised antisymmetrised products of $k$ Bargmann vectors $g^{i_{1} i_{2} \ldots i_{k}}$ defined similarly to $(2.6 b)$ are $\left\{1_{k} \dot{0}\right\}$ shift tensors with shift properties $\Delta^{(k)}\left(i_{1}, i_{2}, \ldots, i_{k}\right)$ when forced to act between $\mathrm{U}(n-1) \times \mathrm{U}(n)$ states of highest weight in their upper patterns.

Although satisfying from the point of view of duplicating the shift properties of the abstract set of tensors of Biedenharn and Louck in a Bargmann representation space, the above prescription severely curtails the possible useful exploitation of the group of upper transformations. We therefore adopt in this investigation a somewhat less restrictive procedure by using the fact that, contrary to Biedenharn and Louck who did not impart a group theoretical meaning to the upper pattern, we can impose such a functional meaning on the upper pattern. More precisely, we require the upper pattern to reflect the tensorial properties of a Bargmann tensor under the $U(n-1)$ complementary group action, the complementarity theorem providing the necessary justification for this prescription. It is then easy to see that the shift properties of an unnormalised and antisymmetrised Bargmann tensor of the form

$$
T\left\langle\left\{1_{k} \dot{0}\right\}\right\rangle(g)=g \wedge g \wedge \ldots \wedge g \quad(k \text { times })
$$

with now an unspecified $U(n-1)$ upper weight can be enforced by coupling this tensor and the initial representation to the desired final representation using only the (multiplicity-free) upper group action.

The construction (2.11) is immediate for tensors of $U(n)$ character $\left\{1_{k} \dot{0}\right\}$ with $\Delta_{n}=0$ but would lead to difficulties for $U(n-1) \times U(n)$ tensors of the type

$$
\mathscr{T}\left(\begin{array}{c}
\left\{1_{k-1} \dot{0}\right\}_{n-1} \\
\left\{1_{k} \dot{0}\right\}_{n} \\
\{m\}_{n-1}
\end{array}\right)
$$

which have $\Delta_{n}=1$. Such tensors would add a box to the $n$th row of the initial tableau which is impossible in the $\mathrm{U}(n-1) \times \mathrm{U}(n)$ Bargmann space since all Young tableaux of this Hilbert space have at most $n-1$ rows. The way out of this apparent impasse is through the use of the $\operatorname{SU}(n)$ equivalence of Young tableaux with columns of length $n$. By adding a square to row $n$, an operator of type (2.12) would create a tableau which is $\operatorname{SU}(n)$ equivalent to a tableau from which a single column of $n$ squares can be removed. A $\left\{1_{k} \dot{0}\right\}$ tensor, with $i_{k}=n$, which would have added a square to row $n$ is thus equivalent to a tensor which removes $n-k$ squares from rows $i_{1}^{\prime}, i_{2}^{\prime}, \ldots, i_{n-k}^{\prime}$ which are the complements of $i_{1}, i_{2}, \ldots, i_{k}$. Such tensors can now be built from $(n-k)$ antisymmetrical coupled derivative operators in the Bargmann space, where we use the fact that $\partial_{j}^{i}=\partial / \partial g_{j}^{i}$ is an annihilation operator for $g_{j}^{i}$. From a construction point of view, we thus subtract from all entries of the double pattern (2.12), with $m_{n n}$ initially set to zero, the non-vanishing $\Delta_{n}$ value so as to bring its new $\Delta_{n}^{\prime}$ value to vanish. This unambiguously prescribes the $\mathrm{U}(n-1) \times \mathrm{U}(n)$ tensorial properties of the Bargmann tensors (2.12) which are thus reset to

$T\left(\begin{array}{c}\left\{\dot{1}_{k-1} \dot{0}_{n-k}\right\}_{n-1} \\ \left\{\dot{1}_{k} \dot{0}_{n-k}\right\}_{n} \\ \{m\}_{n}\end{array}\right)(g) \rightarrow T\left(\begin{array}{c}\left\{\dot{0}_{k-1}-\dot{1}_{n-k}\right\}_{n-1} \\ \left\{\dot{0}_{k}-\dot{1}_{n-k}\right\}_{n} \\ \left\{m-\dot{1}_{n-1}\right\}_{n-1}\end{array}\right)(g) \sim\left(\partial^{1} \wedge \partial^{2} \wedge \ldots \wedge \partial^{n-k}\right)$.

The tensors (2.13) have the right $\mathrm{SU}(n)$ transformation properties and, as required by the Hilbert space structure, vanishing $n$th shift component. 
To conclude this section, we caution the reader not to be induced into thinking that the construction of the Bargmann tensors is straightforward in the most generic case of multiplicity. In fact, except for the multiplicity-free cases and the important case of SU(2) reviewed in part III (Le Blanc 1987), there is still no complete and unambiguous set of rules for their construction as best exemplified by the two substantially different approaches taken by Le Blanc and Rowe $(1986 a, b)$ in regard to the resolution of the $\mathrm{SU}(3)$ multiplicity problem. Work is in progress to try to incorporate the abstract findings of Biedenharn and Louck into the present framework since it is their claim that their approach yields a canonical solution to the $U(n)$ outer multiplicity problem in general.

\section{Reduced Wigner coefficients for the elementary tensors}

The prescription for the computation of the Wigner coefficients follows from the well known Wigner-Eckart theorem. When the shift action of a Bargmann tensor is enforced by the use of an upper $\mathrm{U}(n-1)$ coupling as discussed in $\S 2$, the theorem states that matrix elements of such a coupled Bargmann tensor are, to within normalisation, proportional to $\mathrm{U}(n)$ Wigner coefficients. This prescription has some far-reaching consequences for, recalling that the $\mathrm{U}(n) \downarrow \mathrm{U}(n-1)$ lower decomposition is multiplicity free, we can perform a second $\mathrm{U}(n-1)$ lower coupling of the initial state with the tensor to a desired final $U(n-1)$ lower representation and take the matrix element of the resulting doubly coupled expression with the corresponding final Bargmann state (see (3.1) below). This defines a $\mathrm{U}(n-1) \times \mathrm{U}(n-1)$ reduced matrix element which one immediately concludes to be a $\mathrm{U}(n): \mathrm{U}(n-1)$ reduced Wigner coefficient to within an overall normalisation of all such quantities. In other words, we have reduced the computation of the $\mathrm{U}(n): \mathrm{U}(n-1)$ reduced Wigner coefficients to the computation of $\mathrm{U}(n-1) \times \mathrm{U}(n-1)$ reduced matrix elements of $\mathrm{U}(n-1) \times \mathrm{U}(n)$ Bargmann tensors in a $\mathrm{U}(n-1) \times \mathrm{U}(n)$ Bargmann space. The advantages of such a definite point of view are enormous for actual computations since, when used in conjunction with the powerful vector coherent state theory of $I$, this approach calls upon simple $U(n-1)$ recoupling techniques to supplant the usually tedious boson polynomial manipulations. We thus exploit to their full extent the tensorial properties of the full Bargmann space.

We now proceed with the computation of the reduced Wigner coefficients pertaining to the coupling by the elementary tensors. According to the Wigner-Eckart theorem, we have

$$
\begin{aligned}
& \left\langle\begin{array}{c}
\left\{\boldsymbol{m}^{o}+\Delta^{(k)}(\boldsymbol{i})\right\}_{n-1} \\
\left\{\boldsymbol{m}^{o}+\Delta^{(k)}(\boldsymbol{i})\right\}_{n} \\
\left\{\boldsymbol{m}+\Delta^{(k)}(\boldsymbol{j})\right\}_{n-1}
\end{array}\left\|T\left(\begin{array}{c}
\left\{\dot{\mathbf{1}}_{k} \dot{0}\right\}_{n-1} \\
\left\{\dot{\mathbf{1}}_{k} \dot{0}\right\}_{n} \\
\left\{\dot{1}_{k-1} \varepsilon \dot{0}\right\}_{n-1}
\end{array}\right)\right\|\left\{\begin{array}{c}
\left\{\boldsymbol{m}^{o}\right\}_{n-1} \\
\left\{\boldsymbol{m}^{o}\right\}_{n} \\
\{\boldsymbol{m}\}_{n-1}
\end{array}\right)\right. \\
& =\left\langle\begin{array}{c}
\left\{\boldsymbol{m}^{\circ}+\Delta^{(k)}(\boldsymbol{i})\right\}_{n-1} \\
\left\{m^{o}+\Delta^{(k)}(\boldsymbol{i})\right\}_{n} \\
\left\{m+\Delta^{(k)}(\boldsymbol{j})\right\}_{n-1}
\end{array}\right|\left[T\left(\begin{array}{c}
\left\{\dot{1}_{k} \dot{0}\right\}_{n-1} \\
\left\{\dot{1}_{k} \dot{0}\right\}_{n} \\
\left\{\dot{1}_{k-1} \varepsilon \dot{0}\right\}_{n-1}
\end{array}\right)\left|\begin{array}{c}
\left\{m^{o}\right\}_{n-1} \\
\left\{\boldsymbol{m}^{o}\right\}_{n} \\
\{m\}_{n-1}
\end{array}\right\rangle\right]_{\left\{m+\Delta^{(k)}(j)\right\}_{m-1}}^{\left\{m^{o}+\Delta^{(k)}(i)\right\}_{n-1}} \\
& =\left\langle\begin{array}{c}
\left\{m^{o}\right\}_{n} \\
\{m\}_{n-1}
\end{array} ; \begin{array}{c}
\left\{\dot{1}_{k} \dot{0}\right\}_{n} \\
\left\{\mathbf{1}_{k-1} \varepsilon \dot{0}\right\}_{n-1}
\end{array} \|\left\{\begin{array}{l}
\left\{m^{o}+\Delta^{(k)}(\boldsymbol{i})\right\}_{n} \\
\left\{m+\Delta^{(k)}(\boldsymbol{j})\right\}_{n-1}
\end{array}\right\rangle\right. \\
& \times\left\langle\left\langle\begin{array}{c}
\left\{m^{o}+\Delta^{(k)}(\boldsymbol{i})\right\}_{n-1} \\
\left\{m^{o}+\Delta^{(k)}(\boldsymbol{i})\right\}_{n}
\end{array}\left\|T\left\langle\begin{array}{c}
\left\{\dot{1}_{k} \dot{0}\right\}_{n-1} \\
\left\{\dot{1}_{k} \dot{0}\right\}_{n}
\end{array}\right)\right\|\left\{\begin{array}{c}
\left\{m^{o}\right\}_{n-1} \\
\left\{m^{o}\right\}_{n}
\end{array} \|\right)\right.\right.
\end{aligned}
$$


where the term on the left-hand side of $(3.1 a)$ is the $\mathrm{U}(n-1) \times \mathrm{U}(n-1)$ reduced matrix element of the Bargmann tensor

$$
T\left(\begin{array}{c}
\left\{\mathrm{i}_{k} \dot{0}\right\}_{n-1} \\
\left\{\dot{\mathrm{i}}_{k} \dot{0}\right\}_{n} \\
\left\{\dot{\mathrm{i}}_{k-1} \varepsilon \dot{0}\right\}_{n-1}
\end{array}\right)
$$

and where the square bracket on the right-hand side of $(3.1 a)$ is an (upper $\times$ lower) $\mathrm{U}(n-1) \times \mathrm{U}(n-1)$ coupling $\dagger$ defined in $\S 2.2$ of $\mathrm{I}$. According to the betweenness conditions, $\varepsilon$, in the lower $\mathrm{U}(n-1)$ pattern, is either 0 or 1 .

The first term on the right-hand side of $(3.1 b)$ is the $U(n): U(n-1)$ reduced Wigner coefficient, the quantity which we seek to calculate, while the second term is the total $\mathrm{U}(n-1) \times \mathrm{U}(n)$ doubly reduced matrix element of the tensor. To emphasise that it is doubly reduced with respect to $\mathrm{U}(n-1) \times \mathrm{U}(n)$ and to distinguish it from the $\mathrm{U}(n-1) \times$ $\mathrm{U}(n-1)$ reduced matrix element on the left-hand side of $(3.1 a)$, we have introduced the notation of double bars and double carets.

As for $\Delta^{(k)}(i)=\Delta^{(k)}\left(i_{1}, i_{2}, \ldots, i_{k}\right)($ see $(2.10 a, b))$,

$$
\Delta^{(k)}(j)=\Delta^{(k)}\left(j_{1}, j_{2}, \ldots, j_{k}\right) \quad 1 \leqslant j_{1}<j_{2}, \ldots,<j_{k} \leqslant n
$$

is an $n$-vector indicating the shift affecting the lower $U(n-1)$ partition. Since a $U(n-1)$ partition has only $n-1$ entries, it will be understood that one only considers the first $n-1$ components of $\Delta^{(k)}\left(j_{1}, j_{2}, \ldots, j_{k}\right)$. In the case when its last component is zero ( $j_{k} \neq n$, thus $\varepsilon=1$ ), there is no ambiguity. But for the case $j_{k}=n(\varepsilon=0)$, one must then consider matrix elements of the tensor

$$
\begin{aligned}
& T\left(\begin{array}{c}
\left\{\dot{1}_{k} \dot{0}\right\}_{n-1} \\
\left\{\dot{1}_{k} \dot{0}\right\}_{n} \\
\left\{\dot{1}_{k-1} \dot{0}\right\}_{n-1}
\end{array}\right)\left\langle\begin{array}{c}
\left\{\dot{1}_{k} \dot{0}\right\}_{n-1} \\
\left\{\dot{1}_{k} \dot{0}\right\}_{n} \\
\left\{\dot{1}_{k-1} \dot{0}\right\}_{n-1}
\end{array}\left\|B\left(\begin{array}{c}
\{\dot{0}\}_{n-1} \\
\{1 \dot{0}-1\}_{n} \\
\{\dot{0}-1\}_{n-1}
\end{array}\right)\right\|\left(\begin{array}{c}
\left\{\dot{1}_{k} \dot{0}\right\}_{n-1} \\
\left\{\dot{1}_{k} \dot{0}\right\}_{n} \\
\left\{\dot{1}_{k} \dot{0}\right\}_{n-1}
\end{array}\right)\right. \\
& =\left[B\left(\begin{array}{c}
\{\dot{0}\}_{n-1} \\
\{1 \dot{0}-1\}_{n} \\
\{\dot{0}-1\}_{n-1}
\end{array}\right), T\left(\begin{array}{c}
\left\{\dot{1}_{k} \dot{0}\right\}_{n-1} \\
\left\{\dot{1}_{k} \dot{0}\right\}_{n} \\
\left\{\dot{1}_{k} \dot{0}\right\}_{n-1}
\end{array}\right)\right]_{\left\{i_{k-1} \dot{0}_{n-k}\right\}_{n-1}}
\end{aligned}
$$

obtained from the tensor

$$
T\left(\begin{array}{c}
\left\{\dot{1}_{k} \dot{0}\right\}_{n-1} \\
\left\{\dot{1}_{k} \dot{0}\right\}_{n} \\
\left\{\dot{1}_{k} \dot{0}\right\}_{n-1}
\end{array}\right)
$$

with maximal lower $\mathrm{U}(n-1)$ partition through the action of the lowering operator $\boldsymbol{B}_{i}=E_{n i}$ (see I and note that $\left[E_{n i}, g_{i}^{l}\right]=g_{n}^{l}$ ). The square bracket on the right-hand side of (3.3) is a $U(n-1)$ coupled commutator defined similarly to the coupled expression (2.26) of I while the reduced matrix element multiplying the tensor on the left-hand side is given by $(2.31)$ of $I$.

The total doubly reduced matrix element is given by

$\left\langle\left\langle\begin{array}{c}\left\{m^{o}+\Delta^{(k)}(i)\right\}_{n-1} \\ \left\{m^{o}+\Delta^{(k)}(i)\right\}_{n}\end{array}\left\|T\left\langle\begin{array}{c}\left\{\dot{1}_{k} \dot{0}\right\}_{n-1} \\ \left\{\dot{1}_{k} \dot{0}\right\}_{n}\end{array}\right)\right\|\left\{\begin{array}{c}\left\{m^{o}\right\}_{n-1} \\ \left\{m^{o}\right\}_{n}\end{array}\right)\right\rangle=\frac{N\left(\left\{m^{o}+\Delta^{(k)}(i)\right\}_{n}\right)}{N\left(\left\{m^{o}\right\}_{n}\right)}\right.$

$\dagger$ Upper and lower $U(n-2) \supset U(n-3) \ldots \supset U(1)$ labels are quietly understood for both the bra and the resulting ket in $(3.1 a)$. These labels can assume any allowed values but must match in both bra and resulting ket for the matrix element $(3.1 a)$ not to trivially vanish. 
where $i_{k} \neq n$ and where $N()$ is the normalisation factor (equation (2.7)) for a double Bargmann highest weight state. This result, a weaker version of the boson factorisation lemma (Biedenharn et al 1967) but all that is needed for the problem at hand, is by no means trivial but is nevertheless easily derived within the present framework (Le Blanc and Hecht 1986).

Now, for reasons that will become apparent in the following (see (3.8)), it is easier to consider matrix elements of the Hermitian conjugates of the tensors (3.2) and (3.3). We first consider the case $j_{k} \neq n$ and seek to compute the $\mathrm{U}(n-1) \times \mathrm{U}(n-1)$ reduced matrix element of the Hermitian conjugate of tensor (3.2)

$$
\begin{aligned}
& \left\langle\begin{array}{c}
\left\{m^{o}-\Delta^{(k)}(\boldsymbol{i})\right\}_{n-1} \\
\left\{m^{o}-\Delta^{(k)}(\boldsymbol{i})\right\}_{n} \\
\left\{m-\Delta^{(k)}(\boldsymbol{j})\right\}_{n-1}
\end{array}\left\|T\left(\begin{array}{c}
\left\{\dot{0}-\dot{\mathbf{1}}_{k}\right\}_{n-1} \\
\left\{\dot{0}-\dot{\mathbf{i}}_{k}\right\}_{n} \\
\left\{\dot{0}-\dot{\mathbf{1}}_{k}\right\}_{n-1}
\end{array}\right)\right\|\left\{\begin{array}{c}
\left.m^{o}\right\}_{n-1} \\
\left\{m^{o}\right\}_{n} \\
\{\boldsymbol{m}\}_{n-1}
\end{array}\right)\right. \\
& =(-1)^{\phi\left\{\left\{m^{0}\right\}_{n-1}\right)-\phi\left(\left\{\dot{0}-i_{h}\right\}_{n-1}\right\}-\phi\left(\left\{m^{0}-\Delta^{(h)}(i)\right\}_{n-1}\right)} \\
& \times(-1)^{\phi\left(\{m\}_{n-1}\right)-\phi\left(\left\{\dot{0}-\mathrm{i}_{k}\right\}_{n-1}\right)-\phi\left(\left\{m-\Delta^{(k)}(j)\right\}_{n-1}\right)} \\
& \times\left(\frac{\operatorname{dim}\left(\left\{m^{o}\right\}_{n-1}\right)}{\operatorname{dim}\left(\left\{m^{o}-\Delta^{(k)}(i)\right\}_{n-1}\right)}\right)^{1 / 2}\left(\frac{\operatorname{dim}\left(\{m\}_{n-1}\right)}{\operatorname{dim}\left(\left\{m-\Delta^{(k)}(j)\right\}_{n-1}\right)}\right)^{1 / 2} \\
& \times\left(\begin{array}{c}
\left\{m^{o}\right\}_{n-1} \\
\left\{m^{o}\right\}_{n} \\
\{m\}_{n-1}
\end{array}\left\|T\left\langle\begin{array}{c}
\left\{\dot{1}_{k} \dot{0}\right\}_{n-1} \\
\left\{\dot{1}_{k} \dot{0}\right\}_{n} \\
\left\{\dot{1}_{k} \dot{0}\right\}_{n-1}
\end{array}\right)\right\| \begin{array}{c}
\left\{m^{o}-\Delta^{(k)}(\boldsymbol{i})\right\}_{n-1} \\
\left\{m^{o}-\Delta^{(k)}(\boldsymbol{i})\right\}_{n} \\
\left\{m-\Delta^{(k)}(\boldsymbol{j})\right\}_{n-1}
\end{array}\right)
\end{aligned}
$$

which is equal to

$$
\begin{aligned}
& \left.\left\langle\begin{array}{c}
\left\{\boldsymbol{m}^{o}-\Delta^{(k)}(\boldsymbol{i})\right\}_{n-1} \\
\left\{\boldsymbol{m}^{o}-\Delta^{(k)}(\boldsymbol{i})\right\}_{n} \\
\left\{\boldsymbol{m}-\Delta^{(k)}(\boldsymbol{j})\right\}_{n-1}
\end{array}\right|\left[T\left(\begin{array}{c}
\left\{\dot{0}-\mathrm{i}_{k}\right\}_{n-1} \\
\left\{\dot{0}-\mathrm{i}_{k}\right\}_{n} \\
\left\{\dot{0}-\mathrm{i}_{k}\right\}_{n-1}
\end{array}\right) \mid \begin{array}{c}
\left\{\boldsymbol{m}^{o}\right\}_{n-1} \\
\left\{\boldsymbol{m}^{o}\right\}_{n} \\
\{\boldsymbol{m}\}_{n-1}
\end{array}\right)\right]_{\left\{m-\Delta^{(k)}(\boldsymbol{j})\right\}_{n-1}}^{\left\{m^{(n)}-\Delta^{(k)}(\boldsymbol{i})\right\}_{n-1}} \\
& =\left\langle\left\langle\begin{array}{c}
\left\{m^{o}-\Delta^{(k)}(\boldsymbol{i})\right\}_{n-1} \\
\left\{m^{o}-\Delta^{(k)}(\boldsymbol{i})\right\}_{n}
\end{array}\left\|T\left\langle\begin{array}{c}
\left\{\dot{0}-\dot{1}_{k}\right\}_{n-1} \\
\left\{\dot{0}-\dot{1}_{k}\right\}_{n}
\end{array}\right)\right\|\left\{\begin{array}{c}
\left\{m^{o}\right\}_{n-1} \\
\left\{m^{o}\right\}_{n}
\end{array}\right\rangle\right)\right. \\
& \times\left\langle\begin{array}{cc}
\left\{m^{o}\right\}_{n} & \left.; \dot{0}-\dot{1}_{k}\right\}_{n} \\
\{m\}_{n-1} & \left\{\dot{0}-\dot{1}_{k}\right\}_{n-1}
\end{array} \| \begin{array}{c}
\left\{m^{o}-\Delta^{(k)}(i)\right\}_{n} \\
\left\{m-\Delta^{(k)}(j)\right\}_{n-1}
\end{array}\right\rangle
\end{aligned}
$$

and where, because of the Bargmann space structure, we require $i_{k} \neq n$. This restriction will be lifted below (see (3.13)). We refer the reader to the appendix of I for the definition of the phase $\phi$ and for the various conjugation transformations of Wigner and Racah coefficients used throughout this paper.

The total doubly reduced matrix element is now given by

$$
\begin{aligned}
&\left\langle\left\langle\begin{array}{c}
\left\{m^{o}-\Delta^{(k)}(i)\right\}_{n-1} \\
\left\{m^{o}-\Delta^{(k)}(i)\right\}_{n}
\end{array}\left\|T\left\langle\begin{array}{c}
\left\{\dot{0}-\dot{1}_{k}\right\}_{n-1} \\
\left\{\dot{0}-\dot{1}_{k}\right\}_{n}
\end{array}\right)\right\|\left\{\begin{array}{c}
\left.m^{o}\right\}_{n-1} \\
\left\{m^{o}\right\}_{n}
\end{array}\right)\right\rangle\right. \\
&=(-1)^{\phi\left(\left\{m^{\prime \prime}\right\}_{n-1}\right)-\phi\left(\left\{\dot{0}-\mathrm{i}_{k}\right\}_{n-1}\right)-\phi\left(\left\{m^{\prime \prime}-\Delta^{(k)}(i)\right\}_{n-1}\right)} \\
& \\
& \times(-1)^{\phi\left(\left\{m^{\prime \prime}\right\}_{n}\right)-\phi\left(\left\{0-\dot{i}_{k}\right\}_{n}\right)-\phi\left(\left\{m^{o \prime}-\Delta^{(k)}(i)\right\}_{n}\right)} \\
& \times\left(\frac{\operatorname{dim}\left(\left\{m^{o}\right\}_{n-1}\right)}{\operatorname{dim}\left(\left\{m^{o}-\Delta^{(k)}(i)\right\}_{n-1}\right)}\right)^{1 / 2}\left(\frac{\operatorname{dim}\left(\left\{m^{o}\right\}_{n}\right)}{\operatorname{dim}\left(\left\{m^{o}-\Delta^{(k)}(i)\right\}_{n}\right)}\right)^{1 / 2} \\
& \times \frac{N\left(\left\{m^{o}\right\}_{n}\right)}{N\left(\left\{m^{o}-\Delta^{(k)}(i)\right\}_{n}\right)}
\end{aligned}
$$


through the use of simple conjugation transformations for both $\mathrm{U}(n-1)$ and $\mathrm{U}(n)$ (appendix of $\mathrm{I}$ ) and of (3.4).

Now, from equation (2.26) of I, we can re-express the ket in $(3.5 b)$ in terms of its corresponding highest weight and a polynomial in the lowering operator $B$, and we thus rewrite the coupled expression in $(3.5 b)$ as

$$
\begin{aligned}
& {\left[T\left(\begin{array}{c}
\left\{\dot{0}-\dot{1}_{k}\right\}_{n-1} \\
\left\{\dot{0}-\dot{1}_{k}\right\}_{n} \\
\left\{\dot{0}-\dot{1}_{k}\right\}_{n-1}
\end{array}\right)\left(\begin{array}{c}
\left\{m^{o}\right\}_{n-1} \\
\left\{m^{o}\right\}_{n} \\
\{m\}_{n-1}
\end{array}\right)\right]_{\left\{m-\Delta^{(h)}(j)\right\}_{n-1}}^{\left\{m^{\prime \prime}-\Delta^{(h)}(i)\right\}_{n-1}}} \\
& =(-1)^{\phi\left\{\left\{m^{n}\right\}_{n-1}\right\}-\phi\left(\{\dot{0}-w\}_{n-1}\right\}-\phi\left(\{m\}_{n-1}\right\}} K\left(\begin{array}{c}
\left\{m^{o}\right\}_{n} \\
\{m\}_{n-1}
\end{array}\right)^{-1}\left[\begin{array}{c}
\left\{\dot{0}-\dot{1}_{k}\right\}_{n-1} \\
\left\{\dot{0}-\dot{1}_{k}\right\}_{n} \\
\left\{\dot{0}-\dot{1}_{k}\right\}_{n-1}
\end{array}\right) \\
& \left.\times\left[Z\left(\begin{array}{c}
\{\dot{0}\}_{n-1} \\
\{w \dot{0}-w\}_{n} \\
\{\dot{0}-w\}_{n-1}
\end{array}\right)(B)\left(\begin{array}{c}
\left\{m^{o}\right\}_{n-1} \\
\left\{m^{o}\right\}_{n} \\
\left\{m^{o}\right\}_{n-1}
\end{array}\right)\right]_{\{m\}_{n-1}}^{\left\{m^{n}\right\}_{n-1}}\right]_{\left\{m-\Delta^{(k)}(j)\right\}_{n-1}}^{\left\{m^{n}-\Delta^{(k)}(i)\right\}_{n-1}}
\end{aligned}
$$

Since the commutator

$$
\left.\left[\begin{array}{c}
\left\{\dot{0}-\dot{1}_{k}\right\}_{n-1} \\
\left\{\dot{0}-\dot{1}_{k}\right\}_{n} \\
\left\{\dot{0}-\dot{1}_{k}\right\}_{n-1}
\end{array}\right), Z\left(\begin{array}{c}
\{\dot{0}\}_{n-1} \\
\{w \dot{0}-w\}_{n} \\
\{\dot{0}-w\}_{n-1}
\end{array}\right)(B)\right]=0
$$

vanishes, we can perform a lower $\mathrm{U}(n-1)$ recoupling in (3.7). We then obtain

$$
\begin{aligned}
& \left.\left.T\left(\begin{array}{c}
\left\{\dot{0}-\dot{1}_{k}\right\}_{n-1} \\
\left\{\dot{0}-\dot{1}_{k}\right\}_{n} \\
\left\{\dot{0}-\dot{1}_{k}\right\}_{n-1}
\end{array}\right) \mid \begin{array}{c}
\left\{m^{o}\right\}_{n-1} \\
\left\{m^{o}\right\}_{n} \\
\{m\}_{n-1}
\end{array}\right)\right]_{\left\{m-\Delta^{(k)}(j)\right\}_{n-1}}^{\left\{m^{o}-\Delta^{(k)}(i)\right\}_{n-1}} \\
& =(-1)^{\phi\left(\left\{m^{\prime \prime}-\Delta^{(\alpha)}(i)\right\}_{n-1}-\phi\left(\{\dot{0}-w\}_{n-1}\right)-\phi\left(\left\{m-\Delta^{(\alpha)}(j)\right\}_{n-1}\right)\right.} U\left(\{\dot{0}-w\}\left\{m^{o}\right\}\right. \\
& \left.\times\left\{m-\Delta^{(k)}(j)\right\}\left\{\dot{0}-\dot{1}_{k}\right\} ;\{m\}\left\{m^{o}-\Delta^{(k)}(i)\right\}\right) K\left(\begin{array}{c}
\left\{m^{o}\right\}_{n} \\
\{m\}_{n-1}
\end{array}\right)^{-1} \\
& \times\left[Z ( \begin{array} { c } 
{ \{ \dot { 0 } \} _ { n - 1 } } \\
{ \{ w \dot { 0 } - w \} _ { n } } \\
{ \{ \dot { 0 } - w \} _ { n - 1 } }
\end{array} ) ( B ) \left[T\left(\begin{array}{c}
\left\{\dot{0}-\dot{1}_{k}\right\}_{n-1} \\
\left\{\dot{0}-\dot{1}_{k}\right\}_{n} \\
\left\{\dot{0}-\dot{1}_{k}\right\}_{n-1}
\end{array}\right)\right.\right. \\
& \left.\left.\times\left[\begin{array}{c}
\left\{m^{o}\right\}_{n-1} \\
\left\{m^{o}\right\}_{n} \\
\left\{m^{o}\right\}_{n-1}
\end{array}\right]\right]_{\left\{m^{\prime \prime}-\Delta^{(h)}(\mathbf{i})\right\}_{n-1}}^{\left\{m^{\prime \prime}-\Delta^{(h)}(i)\right\}_{n-1}}\right]_{\left\{m-\Delta^{(h)}(j)\right\}_{n-1}}^{\left\{m^{\prime \prime}-\Delta^{(h)}(i)\right\}_{n-1}} .
\end{aligned}
$$

Note that no sum is required in the recoupling since the betweenness conditions of the double Gel'fand pattern restrict the last entry of the $\mathrm{U}(n-1)$ Racah coefficient

$$
\begin{aligned}
U\left(\{\dot{0}-w\}\left\{m^{o}\right\}\left\{m-\Delta^{(k)}(j)\right\}\left\{\dot{0}-\dot{\mathfrak{i}}_{k}\right\} ;\{m\}\left\{m^{o}-\Delta^{(k)}(\boldsymbol{i})\right\}\right) \\
=U\left(\{w \dot{0}\}\left\{m-\Delta^{(k)}(j)\right\}\left\{m^{o}\right\}\left\{\dot{1}_{k} \dot{0}\right\} ;\left\{m^{o}-\Delta^{(k)}\right\}\{m\}\right)
\end{aligned}
$$

to $\left\{m^{\circ}-\Delta^{(k)}(i)\right\}$. Now, since the tensor

$$
T\left(\begin{array}{c}
\left\{\dot{0}-\dot{1}_{k}\right\}_{n-1} \\
\left\{\dot{0}-\dot{1}_{k}\right\}_{n} \\
\left\{\dot{0}-\dot{1}_{k}\right\}_{n-1}
\end{array}\right)
$$


is a $U(n-1) \times U(n-1)$ tensor when acting on the highest weight state and since the latter can also be considered as a $\mathrm{U}(n-1) \times \mathrm{U}(n-1)$ Bargmann state, we obtain once more from (3.4) and the appendix of $I$ that

$$
\begin{aligned}
& \left.\left[T\left(\begin{array}{c}
\left\{\dot{0}-\mathrm{i}_{k}\right\}_{n-1} \\
\left\{\dot{0}-\dot{1}_{k}\right\}_{n} \\
\left\{\dot{0}-\dot{1}_{k}\right\}_{n-1}
\end{array}\right) \mid \begin{array}{c}
\left\{m^{o}\right\}_{n-1} \\
\left\{m^{o}\right\}_{n} \\
\left\{m^{o}\right\}_{n-1}
\end{array}\right)\right]_{\left\{m^{\prime \prime}-\Delta^{(k)}(i)\right\}_{n-1}}^{\left\{m^{\prime \prime}-\Delta^{(k)}(i)\right\}_{n-1}} \\
& =(-1)^{2\left(\phi\left\{\left\{m^{\prime \prime}\right\}_{n-1}\right)-\phi\left(\left\{\dot{0}-i_{k}\right\}_{n-i}\right)-\phi\left(\left\{m^{\prime \prime}-\Delta^{(k)}(i)\right\}_{n-1}\right)\right)} \\
& \times \frac{N\left(\left\{m^{o}\right\}_{n}\right)}{N\left(\left\{m^{o}-\Delta^{(k)}(i)\right\}_{n}\right.} \frac{\operatorname{dim}\left(\left\{m^{o}\right\}_{n-1}\right)}{\operatorname{dim}\left(\left\{m^{o}-\Delta^{(k)}(i)\right\}_{n-1}\right)} \\
& \left.\times \mid \begin{array}{c}
\left\{m^{o}-\Delta^{(k)}(i)\right\}_{n-1} \\
\left\{m^{o}-\Delta^{(k)}(i)\right\}_{n} \\
\left\{m^{o}-\Delta^{(k)}(i)\right\}_{n-1}
\end{array}\right\} .
\end{aligned}
$$

When (3.9) and (3.10) are introduced in (3.5) and using the expansion (2.26) of I once more, we finally obtain

$$
\begin{aligned}
& \left\langle\begin{array}{cc}
\left\{m^{o}\right\}_{n} & \left\{\dot{0}-\dot{1}_{k}\right\}_{n} \\
\{m\}_{n-1} & \left\{\dot{0}-\dot{1}_{k}\right\}_{n-1}
\end{array} \| \begin{array}{c}
\left\{m^{o}-\Delta^{(k)}(i)\right\}_{n} \\
\left\{m-\Delta^{(k)}(j)\right\}_{n-1}
\end{array}\right\rangle \\
& =(-1)^{\phi\left(\left\{m^{\prime \prime}\right\}_{n}\right)-\phi\left(\left\{\dot{0}-\mathrm{i}_{k}\right\}_{n}\right)-\phi\left(\left\{m^{\prime \prime}-\Delta^{(k)}(i)\right\}_{n}\right\}} \\
& \times(-1)^{\phi\left(\left\{m^{o}\right\}_{n-1}\right)-\phi\left(\left\{0-\mathrm{i}_{k}\right\}_{n-1}\right)-\phi\left(\left\{m^{n}-\Delta^{(k)}(i)\right\}_{n-1}\right)} \\
& \times U\left(\{w \dot{0}\}\left\{m-\Delta^{(k)}(\boldsymbol{j})\right\}\left\{m^{o}\right\}\left\{\dot{1}_{k} \dot{0}\right\} ;\left\{m^{o}-\Delta^{(k)}(\boldsymbol{i})\right\}\{m\}\right) \\
& \times\left(\frac{\operatorname{dim}\left(\left\{m^{o}-\Delta^{(k)}(i)\right\}_{n}\right)}{\operatorname{dim}\left(\left\{m^{o}-\Delta^{(k)}(i)\right\}_{n-1}\right)} \frac{\operatorname{dim}\left(\left\{m^{o}\right\}_{n-1}\right)}{\operatorname{dim}\left(\left\{m^{o}\right\}_{n}\right)}\right)^{1 / 2} \\
& \times K\left(\begin{array}{c}
\left\{m^{o}-\Delta^{(k)}(i)\right\}_{n} \\
\left\{m-\Delta^{(k)}(j)\right\}_{n-1}
\end{array}\right) K\left(\begin{array}{c}
\left\{m^{o}\right\}_{n} \\
\{m\}_{n-1}
\end{array}\right)^{-1} .
\end{aligned}
$$

Using equations (2.33) of I for the dimension factors, (2.23) of I for the $K$ factors, (A2) of I for the phase factors and (A27) and (A28) for the Racah coefficient, (3.11a) then has the explicit expression

$$
\begin{aligned}
& \left\langle\begin{array}{c}
\left\{m^{o}\right\}_{n} \\
\{m\}_{n-1} ;\left\{\dot{0}-\dot{1}_{k}\right\}_{n-1}
\end{array} \| \begin{array}{c}
\left.\left.\dot{0} \dot{i}_{k}\right\}_{n} \| m^{o}-\Delta^{(k)}(i)\right\}_{n} \\
\{m-(j)\}_{n-1}
\end{array}\right) \\
& =(-1)^{\sum_{s=1}^{k}\left(j_{s}-i_{s}\right)}(-1)^{k(k-1) / 2} \prod_{s, t=1}^{k} S\left(j_{s}-i_{r}\right)\left[\prod_{s=1}^{k}\left(\prod_{\substack{i=1 \\
i \neq\left(i_{1}, i_{2}, \ldots, i_{k}\right)}}^{n} \frac{p_{i n}-p_{j_{s}, n-1}}{p_{i n}-p_{i_{s}, n}}\right)\right. \\
& \left.\quad \times\left(\prod_{\substack{j=1 \\
j \neq\left(j_{1}, j_{2}, \ldots, j_{k}\right)}}^{n-1} \frac{p_{j, n-1}-p_{i_{s}, n}+1}{p_{j, n-1}-p_{j_{s}, n-1}+1}\right)\right]^{1 / 2}:
\end{aligned}
$$

The phase in ( $3.11 a)$ can be shown to be equal to $k(k+1)$, i.e. it is always even. Thus the phase factor in $(3.11 b)$ is strictly associated with the phase of the $U(n-1)$ Racah coefficient (see (A12) and (A27)). Also note that, here and in the following, we normalise our results so that they can apply to the cases where the initial representation is a generic $\mathrm{U}(n)$ representation, i.e. when $m_{n n}$ is now a generally non-vanishing quantity unlike the case valid for the $\mathrm{U}(n-1) \times \mathrm{U}(n)$ Bargmann polynomials. Such a 
normalisation is easy to perform by subtracting the quantity $p_{n n}=m_{n n}$ from all the $p_{i j}$ terms originating from the various factors in the basic formula $(3.11 a)$.

Complex conjugation gives us the reduced Wigner coefficient

$$
\begin{aligned}
& \left\langle\begin{array}{cc}
\left\{m^{o}\right\}_{n} & \left\{\dot{1}_{k} \dot{0}\right\}_{n} \\
\{m\}_{n-1} & \left\{\dot{1}_{k} \dot{0}\right\}_{n-1}
\end{array} \|\left\{\begin{array}{c}
\left\{m^{o}+\Delta^{(k)}(\boldsymbol{i})\right\}_{n} \\
\left\{m+\Delta^{(k)}(\boldsymbol{j})\right\}_{n-1}
\end{array}\right\rangle\right. \\
& =(-1)^{\phi\left(\{m\}_{n-1}\right)+\phi\left(\left\{\mathrm{i}_{k} \dot{0}\right\}_{n-1}\right)-\phi\left(\left\{m+\Delta^{(k)}(j)\right\}_{n-1}\right)}(-1)^{\phi\left(\left\{m^{\prime \prime}\right\}_{n}\right)+\phi\left(\left\{\mathrm{i}_{k} 0\right\}_{n}\right)-\phi\left(\left\{m^{\prime \prime}+\Delta^{(k)}(i)\right\}_{n}\right)} \\
& \times\left(\frac{\operatorname{dim}\left(\left\{m^{o}+\Delta^{(k)}(\boldsymbol{i})\right\}_{n}\right.}{\operatorname{dim}\left(\left\{m^{o}\right\}_{n}\right)}\right)^{1 / 2}\left(\frac{\operatorname{dim}\left(\{m\}_{n-1}\right)}{\operatorname{dim}\left(\left\{m+\Delta^{(k)}(j)\right\}_{n-1}\right)}\right)^{1 / 2} \\
& \times\left\langle\begin{array}{cc|c}
\left\{m^{o}+\Delta^{(k)}(\boldsymbol{i})\right\}_{n} & \left\{\dot{0}-\dot{1}_{k}\right\}_{n} & \|\left\{m^{o}\right\}_{n} \\
\left\{m+\Delta^{(k)}(\boldsymbol{j})\right\}_{n-1} & \left\{\dot{0}-\dot{1}_{k}\right\}_{n-1} \|\{m\}_{n-1}
\end{array}\right\rangle \\
& =(-1)^{\phi\left(\left\{m^{o}+\Delta^{(k)}(i)\right\}_{n}\right)-\phi\left(\left\{m^{0}\right\}_{n}\right)+\phi\left(\{m\}_{n-1}\right)-\phi\left(\left\{m+\Delta^{(k)}(j)\right\}_{n-1}\right)-k / 2} \\
& \times U\left(\{w \dot{0}\}\{m\}\left\{m^{o}+\Delta^{(k)}(\boldsymbol{i})\right\}\left\{\dot{\boldsymbol{1}}_{k} \dot{0}\right\} ;\left\{m^{\circ}\right\}\left\{m+\Delta^{(k)}(\boldsymbol{j})\right\}\right) \\
& \times\left(\frac{\operatorname{dim}\left(\left\{m^{o}+\Delta^{(k)}(i)\right\}_{n-1}\right)}{\operatorname{dim}\left(\left\{m+\Delta^{(k)}(j)\right\}_{n-1}\right)} \frac{\operatorname{dim}\left(\{m\}_{n-1}\right)}{\operatorname{dim}\left(\left\{m^{o}\right\}_{n-1}\right)}\right)^{1 / 2} \\
& \times K\left(\begin{array}{c}
\left\{m^{o}\right\}_{n} \\
\{m\}_{n-1}
\end{array}\right) K\left(\begin{array}{c}
\left\{m^{o}+\Delta^{(k)}(i)\right\}_{n} \\
\left\{m+\Delta^{(k)}(j)\right\}_{n-1}
\end{array}\right)^{-1} \\
& =(-1)^{k(k-1) / 2} \prod_{s, t=1}^{k} S\left(j_{s}-i_{t}\right)\left[\prod_{s=1}^{k}\left(\prod_{\substack{i=1 \\
i \neq\left(i_{1}, i_{2}, \ldots, i_{k}\right)}}^{n} \frac{p_{j_{s}, n-1}-p_{i n}+1}{p_{i_{s} n}-p_{i n}}\right)\right. \\
& \left.\times\left(\prod_{j \neq 1}^{n-1} \frac{p_{i_{s}, n}-p_{j, n-1}}{p_{\left.j_{1}, j_{2}, \ldots, j_{k}\right)}-p_{j, n-1}+1}\right)\right]^{1 / 2} .
\end{aligned}
$$

Defining the root conjugate of $\left\{\dot{i}_{k} \dot{0}\right\}_{n}$ by $\left\{\dot{i}_{n-k} 0\right\}_{n}$ and the Hermitian conjugate of $\left\{\mathrm{i}_{k} \dot{0}\right\}_{n}$ by $\left\{\dot{0}-\dot{\mathrm{i}}_{k}\right\}_{n}$, we will, for the case $i_{k}=n$, use the Hermitian conjugate tensor of the root conjugate of the original $\left\{\dot{i}_{k} \dot{0}\right\}_{n}$ tensor to compute the required reduced Wigner coefficient (see $(2.13)$ ). We thus require

$$
\begin{aligned}
& \left\langle\begin{array}{cc}
\left\{\boldsymbol{m}^{o}\right\}_{n} & \left\{\dot{1}_{k} \dot{0}\right\}_{n} \\
\{m\}_{n-1} & \left\{\dot{1}_{k} \dot{0}\right\}_{n-1}
\end{array} \| \begin{array}{c}
\left\{\boldsymbol{m}^{o}+\Delta^{(k)}(\boldsymbol{i})\right\}_{n} \\
\left\{m+\Delta^{(k)}(\boldsymbol{j})\right\}_{n-1}
\end{array}\right\rangle \\
& =\left\langle\begin{array}{cc}
\left\{m^{o}\right\}_{n} & \left\{\dot{0}_{k}-\dot{1}_{n-k}\right\}_{n} \\
\{m\}_{n-1} & \left\{\dot{0}_{k-1}-\dot{1}_{n-k}\right\}_{n-1}
\end{array} \|\left\{\begin{array}{l}
\left\{m^{o}-\Delta^{(k)}\left(\boldsymbol{i}^{\prime}\right)\right\}_{n} \\
\left.(k)\left(j^{\prime}\right)\right\}_{n-1}
\end{array}\right\rangle\right.
\end{aligned}
$$

where the complements $\left(\boldsymbol{i}^{\prime}\right)$ of $(\boldsymbol{i})$ and $\left(\boldsymbol{j}^{\prime}\right)$ of $(\boldsymbol{j})$ are defined such that, for example,

$$
\left\{i_{1}, i_{2}, \ldots, i_{k}\right\}+\left\{i_{1}^{\prime}, i_{2}^{\prime}, \ldots, i_{n-k}^{\prime}\right\}=\{1,2, \ldots, n\} .
$$

We then find, using the phase identity

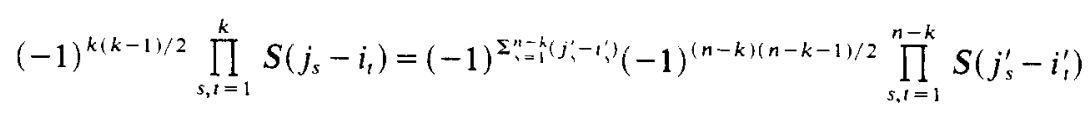

and after an appropriate change of variables, that (3.13) shares the same expression with $(3.12 c)$. Equation $(3.12 c)$ is thus valid for the case $i_{k}=n$. Conversely, $(3.11 b)$ is now assumed valid for $i_{k}=n$. 
When $j_{k}=n$, we have to consider the matrix elements of the tensor (see (3.3))

$$
\begin{aligned}
& T\left(\begin{array}{c}
\left\{\dot{0}-\dot{1}_{k}\right\}_{n-1} \\
\left\{\dot{0}-\dot{1}_{k}\right\}_{n} \\
\left\{\dot{0}-\dot{1}_{k-1}\right\}_{n-1}
\end{array}\right)\left\langle\begin{array}{c}
\left\{\dot{0}-\dot{1}_{k}\right\}_{n-1} \\
\left\{\dot{0}-\dot{1}_{k}\right\}_{n} \\
\left\{\dot{0}-\dot{1}_{k-1}\right\}_{n-1}
\end{array}\left\|A\left(\begin{array}{c}
\{\dot{0}\}_{n-1} \\
\{1 \dot{0}-1\}_{n} \\
\{1 \dot{0}\}_{n-1}
\end{array}\right)\right\|\left(\begin{array}{c}
\left\{\dot{0}-\dot{1}_{k}\right\}_{n-1} \\
\left\{\dot{0}-\dot{1}_{k}\right\}_{n} \\
\left\{\dot{0}-\dot{1}_{k}\right\}_{n-1}
\end{array}\right)\right. \\
& =(-1)\left[A\left(\begin{array}{c}
\{\dot{0}\}_{n-1} \\
\{1 \dot{0}-1\}_{n} \\
\{1 \dot{0}\}_{n-1}
\end{array}\right), T\left(\begin{array}{c}
\left\{\dot{0}-\dot{1}_{k}\right\}_{n-1} \\
\left\{\dot{0}-\dot{1}_{k}\right\}_{n} \\
\left\{\dot{0}-\dot{1}_{k}\right\}_{n-1}
\end{array}\right)\right]_{\left\{0-i_{k-1}\right\}_{n-1}}
\end{aligned}
$$

where the commutator in $(3.14)$ is a lower $\mathrm{U}(n-1)$ coupled commutator (the upper coupling being trivial) and where the reduced matrix element

$$
\left(\begin{array}{c}
\left\{\dot{0}-\dot{1}_{k}\right\}_{n-1} \\
\left\{\dot{0}-\dot{1}_{k}\right\}_{n} \\
\left\{\dot{0}-\dot{1}_{k-1}\right\}_{n-1}
\end{array}\left\|A\left(\begin{array}{c}
\{\dot{0}\}_{n-1} \\
\{1 \dot{0}-1\}_{n} \\
\{1 \dot{0}\}_{n-1}
\end{array}\right)\right\|\left\{\begin{array}{c}
\left.\dot{0}-\dot{1}_{k}\right\}_{n-1} \\
\left\{\dot{0}-\dot{1}_{k}\right\}_{n} \\
\left\{\dot{0}-\dot{1}_{k}\right\}_{n-1}
\end{array}\right)=\left(\frac{\operatorname{dim}\left(\left\{\dot{0}-\dot{1}_{k}\right\}_{n-1}\right)}{\operatorname{dim}\left(\left\{\dot{0}-\dot{1}_{k-1}\right\}_{n-1}\right)}\right)^{1 / 2}[k]^{1 / 2}\right.
$$

is obtained from (2.32) of $I$.

Applying the coupled commutator on a double Gel'fand state, expanding and recoupling, we obtain

$$
\begin{aligned}
& \left.\left.\left[\left[\begin{array}{c}
\{\dot{0}\}_{n-1} \\
\{1 \dot{0}-1\}_{n} \\
\{1 \dot{0}\}_{n-1}
\end{array}\right), T\left(\begin{array}{c}
\left\{\dot{0}-\dot{1}_{k}\right\}_{n-1} \\
\left\{\dot{0}-\dot{1}_{k}\right\}_{n} \\
\left\{\dot{0}-\dot{1}_{k}\right\}_{n-1}
\end{array}\right)\right]_{\left\{\dot{0}-i_{k-1}\right\}_{n-1}}^{\left\{\dot{0}-\dot{i}_{k}\right\}_{n-1}} \mid \begin{array}{c}
\left\{m^{o}\right\}_{n-1} \\
\left\{m^{o}\right\}_{n} \\
\{m\}_{n-1}
\end{array}\right)\right]_{\left\{m-\Delta^{(k)}(j)\right\}_{n-1}}^{\left\{m^{o}-\Delta^{(k)}(i)\right\}_{n-1}} \\
& =\sum_{\left\{m^{\prime}\right\}} U\left(\{m\}\left\{\dot{0}-\dot{1}_{k}\right\}\left\{m-\Delta^{(k)}(j)\right\}\{1 \dot{0}\} ;\left\{m^{\prime}\right\}\left\{\dot{0}-\dot{1}_{k-1}\right\}\right) \\
& \times\left(\begin{array}{c}
\left\{m^{o}-\Delta^{(k)}(\boldsymbol{i})\right\}_{n-1} \\
\left\{m^{o}-\Delta^{(k)}(\boldsymbol{i})\right\}_{n} \\
\left\{m-\Delta^{(k)}(\boldsymbol{j})\right\}_{n-1}
\end{array}\left\|A\left(\begin{array}{c}
\{\dot{0}\}_{n-1} \\
\{1 \dot{0}-1\}_{n} \\
\{1 \dot{0}\}_{n-1}
\end{array}\right)\right\|\left\{\begin{array}{c}
\left\{m^{o}-\Delta^{(k)}(\boldsymbol{i})\right\}_{n-1} \\
\left\{m^{o}-\Delta^{(k)}(\boldsymbol{i})\right\}_{n} \\
\left\{\boldsymbol{m}^{\prime}\right\}_{n-1}
\end{array}\right)\right. \\
& \times\left[T\left(\begin{array}{c}
\left\{\dot{0}-\dot{1}_{k}\right\}_{n-1} \\
\left\{\dot{0}-\dot{1}_{k}\right\}_{n} \\
\left\{\dot{0}-\dot{1}_{k}\right\}_{n-1}
\end{array}\right)\left(\begin{array}{c}
\left\{m^{o}\right\}_{n-1} \\
\left\{m^{o}\right\}_{n} \\
\{m\}_{n-1}
\end{array}\right)\right]_{\left\{m^{\prime}\right\}_{n-1}}^{\left\{m^{\prime \prime}-\Delta^{(k)}(i)\right\}_{n-1}} \\
& -\sum_{\left\{m^{\prime \prime}\right\}}(-1)^{\phi\left(\left\{\dot{0}-i_{k}\right\}_{n-1}\right)-\phi\left(\{10\}_{n-1}\right)-\phi\left(\left\{0-i_{k-1}\right\}_{n-1}\right)} \\
& \times U\left(\{m\}\{1 \dot{0}\}\left\{m-\Delta^{(k)}(j)\right\}\left\{\dot{0}-\dot{1}_{k}\right\} ;\left\{m^{\prime \prime}\right\}\left\{\dot{0}-\dot{1}_{k-1}\right\}\right. \\
& \times\left[T\left(\begin{array}{c}
\left\{\dot{0}-\dot{1}_{k}\right\}_{n-1} \\
\left\{\dot{0}-\dot{1}_{k}\right\}_{n} \\
\left\{\dot{0}-\dot{1}_{k}\right\}_{n-1}
\end{array}\right)\left(\begin{array}{c}
\left\{m^{o}\right\}_{n-1} \\
\left\{m^{o}\right\}_{n} \\
\left\{m^{\prime \prime}\right\}_{n-1}
\end{array}\right)\right]_{\left\{m-\Delta^{(k)}(j)\right\}_{n-1}}^{\left(m^{\prime \prime}-\Delta^{(k)}(i)\right\}_{n-1}} \\
& \times\left(\begin{array}{c}
\left\{m^{o}\right\}_{n-1} \\
\left\{m^{o}\right\}_{n} \\
\left\{m^{\prime \prime}\right\}_{n-1}
\end{array}\left\|A\left(\begin{array}{c}
\{\dot{0}\}_{n-1} \\
\{1 \dot{0}-1\}_{n} \\
\{1 \dot{0}\}_{n-1}
\end{array}\right)\right\| \begin{array}{c}
\left\{m^{o}\right\}_{n-1} \\
\left\{m^{o}\right\}_{n} \\
\{m\}_{n-1}
\end{array}\right)
\end{aligned}
$$

where, once more, we require $i_{k} \neq n$. Using (A13) and (A14) for the Racah coefficients $U\left(\{m\}\{1 \dot{0}\}\left\{m-\Delta^{(k)}(j)\right\}\left\{\dot{0}-\dot{1}_{k}\right\} ;\left\{m^{\prime}\right\}\left\{\dot{0}-\dot{1}_{k-1}\right\}\right)$

$$
\begin{aligned}
= & (-1)^{\phi\left(\left\{m^{\prime}\right\}_{n-1}\right)+\phi\left(\left\{\dot{0}-\mathrm{i}_{n-1}\right\}_{n-1}-\phi\left(\{m\}_{n-1}\right)-\phi\left(\left\{\dot{0}-\mathrm{i}_{k}\right\}_{n-1}\right)\right.} \\
& \times\left(\frac{\operatorname{dim}\left(\left\{m^{\prime}\right\}_{n-1}\right) \operatorname{dim}\left(\left\{\dot{0}-\dot{1}_{k-1}\right\}_{n-1}\right)}{\operatorname{dim}\left(\{m\}_{n-1}\right) \operatorname{dim}\left(\left\{\dot{0}-\dot{\mathrm{i}}_{k}\right\}_{n-1}\right)}\right)^{1 / 2} \\
& \times U\left(\left\{m-\Delta^{(k)}(j)\right\}\left\{\dot{1}_{k-1} \dot{0}\right\}\left\{m^{\prime}\right\}\{1 \dot{0}\} ;\{m\}\left\{\dot{1}_{k} \dot{0}\right\}\right)
\end{aligned}
$$


and

$$
\begin{aligned}
U\left(\{m\}\left\{\dot{0}-\dot{1}_{k}\right\}\right. & \left.\left\{m-\Delta^{(k)}(j)\right\}\{1 \dot{0}\} ;\left\{m^{\prime \prime}\right\}\left\{\dot{0}-\dot{1}_{k-1}\right\}\right) \\
= & (-1)^{\phi\left(\left\{m^{\prime \prime}\right\}_{n-1}\right)+\phi\left(\left\{\dot{0}-\dot{1}_{k-1}\right\}_{n-1}\right)-\phi\left(\left\{m-\Delta^{(k)}(j)\right\}_{n-1}\right)-\phi\left(\left\{\dot{0}-\dot{1}_{k}\right\}_{n-1}\right)} \\
& \times\left(\frac{\operatorname{dim}\left(\left\{m^{\prime \prime}\right\}_{n-1}\right) \operatorname{dim}\left(\left\{\dot{0}-\dot{1}_{k-1}\right\}_{n-1}\right)}{\operatorname{dim}\left(\left\{m-\Delta^{(k)}(j)\right\}_{n-1}\right) \operatorname{dim}\left(\left\{\dot{0}-\dot{1}_{k}\right\}_{n-1}\right)}\right)^{1 / 2} \\
& \times U\left(\left\{m^{\prime \prime}\right\}\{1 \dot{0}\}\{m\}\left\{\dot{1}_{k-1} \dot{0}\right\} ;\left\{m-\Delta^{(k)}(j)\right\}\left\{\dot{1}_{k} \dot{0}\right\}\right)
\end{aligned}
$$

equation (2.32) of I for the reduced matrix element of $A$, the phase convention of the appendix of $I$ and the preceding results concerning the reduced matrix elements of

$$
T\left(\begin{array}{c}
\left\{\dot{0}-\dot{1}_{k}\right\}_{n-1} \\
\left\{\dot{0}-\dot{1}_{k}\right\}_{n} \\
\left\{\dot{0}-\dot{1}_{k}\right\}_{n-1}
\end{array}\right)
$$

we obtain for the case $j_{k}=n$

$$
\begin{aligned}
&\left\langle\begin{array}{c}
\left\{m^{o}\right\}_{n} \\
\{m\}_{n-1}
\end{array} ; \begin{array}{c}
\left\{\dot{0}-\dot{1}_{k}\right\}_{n} \\
\left\{\dot{0}_{n-k}-\dot{1}_{k-1}\right\}_{n-1}
\end{array} \| \begin{array}{c}
\left\{m^{o}-\Delta^{(k)}(i)\right\}_{n} \\
\left\{m-\Delta^{(k)}(j)\right\}_{n-1}
\end{array}\right\rangle \\
&=\frac{\mathscr{S}}{(n-k)}(-1)^{\Sigma_{s=1}^{k}\left(j_{s}-i_{s}\right)}(-1)^{k(k-1) / 2} \prod_{s, t=1}^{k} S\left(j_{s}-i_{t}\right) \\
& \times\left[\prod_{s=1}^{k}\left(\prod_{\substack{i=1 \\
i \neq\left(i_{1}, i_{2}, \ldots, i_{k}\right)}}^{n} \frac{p_{i n}-p_{j_{s}, n-1}}{p_{i n}-p_{i_{s}, n}}\right)\left(\prod_{\substack{j=1 \\
j \neq\left(j_{1}, j_{2}, \ldots, j_{k}\right)}}^{n-1} \frac{p_{j, n-1}-p_{i_{s}, n}+1}{p_{j, n-1}-p_{j_{s}, n-1}+1}\right)\right]^{1 / 2}
\end{aligned}
$$

where in order to condense the notation, it is understood that one must take the limit $p_{n, n-1} \rightarrow \infty$ in (3.18), therefore ignoring all factors involving this term. $\mathscr{I}$, in (3.18), is a sum given by

$$
\mathscr{S}=\sum_{\substack{j_{a}=1 \\ j_{a} \neq\left(j_{1}, j_{2}, \ldots, j_{k-1}\right)}}^{n-1}\left(\frac{\prod_{i=1, i \neq\left(i_{1}, i_{2}, \ldots, i_{k}\right)}^{n}\left(p_{j_{a}, n-1}-p_{i n}+1\right)-\prod_{i=1, i \neq\left(i_{1}, i_{2}, \ldots, i_{k}\right)}^{n}\left(p_{j_{a}, n-1}-p_{i n}\right)}{\prod_{j=1, j \neq\left(j_{a}, j_{1}, j_{2}, \ldots, j_{k-1}\right)}^{n-1}\left(p_{j_{a}, n-1}-p_{j, n-1}\right)}\right) .
$$

By using contour integration of the complex function

$$
F(z)=\left(\frac{\prod_{i=1, i \neq\left(i_{1}, i_{2}, \ldots, i_{k}\right)}^{n}\left(z-p_{i n}+1\right)-\prod_{i=1, i \neq\left(i_{1}, i_{2}, \ldots, i_{k}\right)}^{n}\left(z-p_{i n}\right)}{\prod_{j=1, j \neq\left(j_{1}, j_{2}, \ldots, j_{k-1}\right)}^{n-1}\left(z-p_{j, n-1}\right)}\right)
$$

it is easy to show that $\mathscr{S}=(n-k)$. We therefore note that with the convention that one must take the limit $p_{n, n-1} \rightarrow \infty$ in (3.18), this last equation has exactly the same functional dependence on the $p$ as $(3.11 b)$. This fact brought Biedenharn and Louck to claim that there is a hook permutational symmetry among the various reduced Wigner coefficients. This claim should be taken with some caution for, although true for the functional dependence of the reduced Wigner coefficients on the $p$, the statement is seen not to apply to $(3.11 a)$. Structurally speaking, $(3.11 a)$ does not generalise to the case $i_{k}, j_{k}=n$ since the $\mathrm{U}(n-1)$ Racah coefficient then loses its significance. 


\section{Summary and discussion}

We have shown that the $\mathrm{U}(n): \mathrm{U}(n-1)$ Wigner coefficients pertaining to the coupling by the elementary tensors are given by the structural expressions $(3.12 b)$ and $(3.11 a)$ valid for

$$
1 \leqslant i_{1}<i_{2}<\ldots<i_{k}<n \quad 1 \leqslant j_{1}<j_{2}<\ldots<j_{k}<n
$$

and generic $\operatorname{SU}(n)\left(m_{n n}=0\right)$ unirreps.

We recall the origin of the various terms in these equations. The terms like $\operatorname{dim}\left(\left\{m^{\circ}\right\}_{n}\right)$ and $\operatorname{dim}\left(\{m\}_{n-1}\right)$ are the dimensions of the respective $\mathrm{U}(n)$ and $\mathrm{U}(n-1)$ representations $\left\{m^{\circ}\right\}_{n}$ and $\{m\}_{n-1}$. The factors $U()$ are simple multiplicity-free $U(n-$ 1) Racah coefficients calculated in the appendix. The terms in $K$ are normalisation factors obtained from the vector coherent state theory of $I$. Note that there are no terms involving $N($ ), the normalisation factor of the Bargmann polynomials (2.7). This is most satisfying since expressions for the Wigner coefficients should not explicitly refer to the space used for their computation.

More generally, lifting the restrictions on $i_{k}$ and $j_{k}$, and more explicitly, we have shown that the $\mathrm{U}(n): \mathrm{U}(n-1)$ Wigner coefficients are given by $(3.12 c)$ and $(3.11 b)$ valid for

$$
1 \leqslant i_{1}<i_{2}<\ldots<i_{k} \leqslant n \quad 1 \leqslant j_{1}<j_{2}<\ldots<j_{k} \leqslant n
$$

and generic $\mathrm{U}(n)\left(m_{n n} \neq 0\right)$ unirreps but where one must take the limit $p_{n, n-1} \rightarrow \infty$.

The final phase factors resulting from the computation correspond to a generalisation of the Condon-Shortley phase convention for $\mathrm{SU}(2)$ and naturally arise within the framework adopted here. We recall that we have introduced only two specific rules for fixing the phases appearing at various computational stages. One pertains to the sign associated with the hooks (see the appendix) and is responsible for the term $S\left(j_{s}-i_{i}\right)$. The other, defined in the appendix of $\mathrm{I}$, is responsible for the conjugation phase

$$
(-1)^{\sum_{r=1}^{\lambda}\left(j_{s}-i\right)} \text {. }
$$

The only phase factor left to consider is

$$
(-1)^{k(k-1) / 2}
$$

and originates from the tensor (antisymmetrical) structure itself.

An interesting point to mention is the fact that the algorithm of $\S 3$ is truly recursive in $n$, i.e. the computation of $\mathrm{U}(n): \mathrm{U}(n-1)$ reduced Wigner coefficients presupposes only the knowledge of $U(n-1)$ Wigner and Racah coefficients for, as shown in Le Blanc and Hecht (1986), the knowledge of the $\mathrm{U}(n-1) \times \mathrm{U}(n)$ normalisation factors (3.4) and (3.6) is a direct consequence of the knowledge of the $\mathrm{U}(n-1) \times \mathrm{U}(n-1)$ reduced matrix element (3.10). Thus, contrary to the approach of Biedenharn and Louck which requires an a priori knowledge of $\mathrm{U}(n) \times \mathrm{U}(n)$ reduced matrix elements in order to allow the computation of the elementary $\mathrm{U}(n): \mathrm{U}(n-1)$ reduced Wigner coefficients (the substance of the boson factorisation lemma), the algorithm of $\S 3$ is much more satisfying on computational grounds since it clearly demonstrates that a $\mathrm{U}(n)$ Wigner-Racah calculus can be performed entirely in a $\mathrm{U}(n-1) \times \mathrm{U}(n-1)$ framework. The extra ( $n$ th) dimension only appears through the application of raising and lowering operators $A$ and $B$ on $U(n-1)$ states and tensors (see (3.14)-(3.19)) and are themselves considered as $\mathrm{U}(n-1)$ tensors (see I). 
Biedenharn and Louck (1968) gave a graphical representation of (3.11) and (3.12c) which, although extremely simple, does not contain the group theoretical information of $(3.11 a)$ and $(3.12 b)$. It is this graphical representation which they coined the "pattern calculus'; the reader is referred to their work for an enunciation of the rules of this calculus.

We have thus duplicated and improved on the results of Biedenharn and Louck since a major result obtained in this paper, which cannot be appreciated from the Biedenharn and Louck mode of derivation, is that, except for the $i_{k}, j_{k}=n$ case, the elementary Wigner coefficients are essentially $\mathrm{U}(n-1)$ Racah coefficients. In fact, comparing equations (A27) and (A28) and (3.11b) and (3.12c), one notices that, except for the contribution of the $i=n$ factors in $(3.11 b)$ and $(3.12 c)$, the Wigner coefficients are $U(n-1)$ Racah coefficients.

We conclude that the combination of two powerful tools, namely the vector coherent state theory and the complementarity principle, in addition to a strict and novel interpretation of the operator patterns, helped us to elucidate the structure of the elementary Wigner-Racah calculus for the $U(n)$ groups and have reduced it to an exercise in recoupling techniques with its foregoing simplicity. Also, since the Racah coefficients required were computed using symmetric group techniques, our results tighten the already known strong relationship between the two groups.

Part III (Le Blanc 1987) of this series shows that the present framework allows the easy computation in closed form of all the SU(2) Wigner coefficients. Also, we generalise the present results to the case of symmetric $\mathrm{U}(n)$ tensors to derive an interesting relationship between $\mathrm{U}(2)$ Racah coefficients and $\mathrm{U}(3)$ symmetric tensors. This relationship, the well known Elliott-Biedenharn-Racah identity but in a new parametrisation, enables us to derive a recursion formula for $\mathrm{U}(2)$ Racah coefficients which is solved to provide a closed form for these coefficients.

Finally, we intend to address in a future work the question of the construction of generic Bargmann tensors in order to see if the present approach would yield a transparent solution to the multiplicity problem.

\section{Appendix. Derivation of multiplicity-free $U(n)$ Racah coefficients using symmetric group techniques}

We compute in this appendix the following classes of multiplicity-free $\mathrm{U}(n)$ Racah coefficients:

$$
\begin{aligned}
& U\left(\{g\}\{1\}\{f\}\{w-1\} ;\left\{g^{\prime}\right\}\{w\}\right) \\
& U\left(\{g\}\{w-1\}\{f\}\{1\} ;\left\{f^{\prime}\right\}\{w\}\right) \\
& U\left(\{g\}\{1\}\{f\}\left\{1_{k-1}\right\} ;\left\{g^{\prime}\right\}\left\{1_{k}\right\}\right) \\
& U\left(\{g\}\left\{1_{k-1}\right\}\{f\}\{1\} ;\left\{f^{\prime}\right\}\left\{1_{k}\right\}\right) \\
& U\left(\{w\}\left\{f_{2}\right\}\{f\}\left\{1_{k}\right\} ;\left\{f_{12}\right\}\left\{f_{23}\right\}\right)
\end{aligned}
$$

where $\{f\}$ is an arbitrary $U(n)$ unirrep and where $\{w\}$ and $\left\{1_{k}\right\}$ are symmetrical and antisymmetrical unirreps, respectively.

The first two classes of coefficients have already been discussed by Kramer in terms of Young frame axial distance expressions (Kramer 1967); the third class follows from a generalisation of results derived in Hecht (1975). 
Consider a system described by a wavefunction

$$
\psi\left(\boldsymbol{r}_{1}, \boldsymbol{r}_{2}, \ldots, \boldsymbol{r}_{N}\right)_{m ; y_{N} y_{N-1} \ldots y_{2} y_{1}}^{\{f}=\left\langle\left.\mathbf{r}\right|_{m ; y_{N} y_{N-1} \ldots y_{2} y_{1}} ^{\{f}\right\rangle
$$

which has for arguments the $\mathrm{Nn}$ variables

$$
r_{s} \equiv r_{s i} \quad s=1, \ldots, N, i=1, \ldots, n
$$

e.g. an $N$-particle system in an $n$-dimensional space. This wavefunction belongs simultaneously to a basis for the $\mathrm{U}(n)$ irreducible unitary representation $\{f\}$ with basis elements conveniently enumerated by a lower Gel'fand pattern $m$ corresponding to the subgroup decomposition

$$
\mathrm{U}(n) \supset \mathrm{U}(n-1) \supset \ldots \supset \mathrm{U}(2) \supset \mathrm{U}(1)
$$

and to a basis for the $S_{N}$ irreducible unitary representation also labelled by $\{f\}$ though the well known complementarity of $\mathrm{U}(n)$ and $S_{N}$ unirreps with basis elements conveniently enumerated by a Yamanouchi symbol $y_{N} y_{N-1} \ldots y_{2} y_{1}$ corresponding to the subgroup decomposition

$$
S_{N} \supset S_{N-1} \supset \ldots \supset S_{2} \supset S_{1} \text {. }
$$

This wavefunction can be expanded as

$$
\begin{gathered}
\psi\left(\boldsymbol{r}_{1}, \ldots, \boldsymbol{r}_{N}\right)_{m ; y_{N} y_{N-1} \ldots y_{2} y_{1}}^{\{f\}}=\left[\left[\ldots\left[\left[\psi\left(\boldsymbol{r}_{1}\right)^{\{1\}} \psi\left(\boldsymbol{r}_{2}\right)^{\{1\}}\right]^{\left\{f^{(2)}\right\}} \psi\left(\boldsymbol{r}_{3}\right)^{\{1\}}\right]^{\left\{f^{(3)}\right\}}\right.\right. \\
\left.\times \ldots \times \psi\left(\boldsymbol{r}_{N-1}\right)^{\{1\}}\right]^{\left\{f^{(N-1)\}} \psi\left(\boldsymbol{r}_{N}\right)^{\{1\}}\right]_{m}^{\left\{f^{(N)}\right\}}}
\end{gathered}
$$

where a square bracket [ ] denotes a $U(n)$ coupling and $\left\{f^{(N-1)}\right\}$ is obtained from $\{f\} \equiv\left\{f^{(N)}\right\}$ by removing one square from the $y_{N}$ th row of the Young tableau representing the partition $\{f\}$.

The transformation to a non-standard representation of $S_{N}$ has been given by Horie (1964) in the special case when the last $w$ (or $k$ ) particles transform under the totally symmetric (totally antisymmetric) representation of $S_{w}\left(S_{k}\right)$. In the symmetric case, the wavefunction is given in a $S_{w} \times\left(S_{N-w} \supset S_{N-w-1} \supset \ldots \supset S_{2} \supset S_{1}\right)$ basis:

$$
\begin{aligned}
\psi\left(\boldsymbol{r}_{1}, \ldots, \boldsymbol{r}_{N}\right)_{m ; y_{N} y_{N-1} \ldots y_{2} y_{1}}^{\{f\}} & =\left[\left[\ldots\left[\left[\psi\left(\boldsymbol{r}_{1}\right)^{\{1\}} \psi\left(\boldsymbol{r}_{2}\right)^{\{1\}}\right]^{\left\{f^{\{2\}}\right.} \psi\left(\boldsymbol{r}_{3}\right)^{\{1\}}\right]^{\{3\}\}} \times \ldots \times \psi\left(\boldsymbol{r}_{N-w}\right)^{\{1\}}\right]^{\left\{f^{\{N-1)\}}\right.}\right. \\
& \left.\times \psi\left(\boldsymbol{r}_{N-w+1}, \ldots, \boldsymbol{r}_{N}\right)^{\{w\}}\right]_{m}^{\{f\}} \\
\equiv & \left\langle\boldsymbol{r}||_{m}^{\{f\}} ;\left\{y_{N} y_{N-1} \ldots y_{N-w+1}\right\}_{\{w\}} y_{N-w} y_{N-w-1} \ldots y_{1}\right\rangle
\end{aligned}
$$

where

$$
\begin{aligned}
&\left|\underset{m}{\{f} ;\left\{b_{1} b_{2} \ldots b_{w}\right\}_{\{w\}} y_{N-w} y_{N-w-1} \ldots y_{1}\right\rangle \\
&=\sum_{i=1}^{w}\left(\frac{1}{w} \prod_{\substack{j=1 \\
j \neq 1}}^{w}\left(1+1 / \tau_{b, b}\right)\right)^{1 / 2} \\
&\left.\times\left.\right|_{m} ^{\{f\}} ;\left\{b_{1} b_{2} \ldots b_{i-1} b_{i+1} \ldots b_{w}\right\}_{\{w-1\}} b_{i} y_{N-w} y_{N-w-1} \ldots y_{1}\right\rangle \\
&= \sum_{i=1}^{w}\left(\frac{1}{w} \prod_{\substack{j=1 \\
j \neq i}}^{w}\left(1+1 / \tau_{b, b}\right)\right)^{1 / 2} \\
&\left.\times\left.\right|_{m} ^{\{f\}} ; b_{i}\left\{b_{1} b_{2} \ldots b_{i-1} b_{i+1} \ldots b_{w}\right\}_{\{w-1\}} y_{N-w} y_{N-w-1} \ldots y_{1}\right\rangle
\end{aligned}
$$

with $\tau_{b, b}$ a Young tableau axial distance to be defined below. 
From (A3a), we have

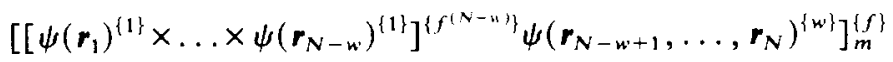

$$
\begin{aligned}
& =\sum_{\left\{f^{(N-n+1)}\right\}}\left(\frac{1}{w} \prod_{\substack{j=1 \\
j \neq i}}^{w}\left(1+1 / \tau_{b, b}\right)\right)^{1 / 2} \\
& \times\left[\left[\left[\psi\left(\boldsymbol{r}_{1}\right)^{\{1\}} \times \ldots \times \psi\left(\boldsymbol{r}_{N-w}\right)^{\{1\}}\right]^{\left\{f^{(N-n)\}}\right.} \psi\left(\boldsymbol{r}_{N-\boldsymbol{w}+1}\right)^{\{1\}}\right]^{\left\{f^{(N-n+1)\}}\right.}\right. \\
& \left.\times \psi\left(\boldsymbol{r}_{N-w+2}, \ldots, \boldsymbol{r}_{N}\right)^{\{w-1\}}\right]_{m}^{\{f\}}
\end{aligned}
$$

where $\left\{f^{(N-w+1)}\right\}$ is obtained from $\left\{f^{(N-w)}\right\}$ by adding one square to the $b_{i}$ th row of the tableau $\left\{f^{(N-w)}\right\}$. Since

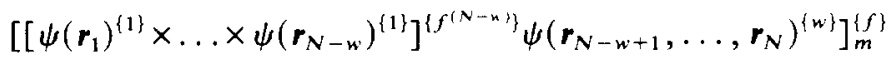

$$
\begin{aligned}
& =\left[\left[\psi\left(\boldsymbol{r}_{1}\right)^{\{1\}} \times \ldots \times \psi\left(\boldsymbol{r}_{N-w}\right)^{\{1\}}\right]^{\left\{f^{(N-n)}\right\}}\right. \\
& \left.\times\left[\psi\left(\boldsymbol{r}_{N-w+1}\right)^{\{1\}} \psi\left(\boldsymbol{r}_{N-w+2}, \ldots, \boldsymbol{r}_{N}\right)^{\{w-1\}}\right]^{\{w\}}\right]_{m}^{\{f\}} \\
& \left.=\sum_{\left\{f^{(N-w+1)}\right\}} U\left\{f^{(N-w)}\right\}\{1\}\{f\}\{w-1\} ;\left\{f^{(N-w+1)}\right\}\{w\}\right) \\
& \times\left[\left[\left[\psi\left(\boldsymbol{r}_{1}\right)^{\{1\}} \times \ldots \times \psi\left(\boldsymbol{r}_{N-w}\right)^{\{1\}}\right]^{\left\{f^{(N-N)}\right.} \psi\left(\boldsymbol{r}_{N-w+1}\right)^{\{1\}}\right]^{\left\{f^{(N-N+1)\}}\right.}\right. \\
& \left.\times \psi\left(\boldsymbol{r}_{N-w+2}, \ldots, \boldsymbol{r}_{N}\right)^{\{w-1\}}\right]_{m}^{\{f\}}
\end{aligned}
$$

comparing with (A4) gives

$$
\begin{gathered}
U\left(\left\{f^{(N-w)}\right\}\{1\}\{f\}\{w-1\} ;\left\{f^{(N-w+1)}\right\}\{w\}\right)=\left(\frac{1}{w} \prod_{\substack{j=1 \\
j \neq i}}^{w}\left(1+1 / \tau_{b, b}\right)\right)^{1 / 2} \\
=U\left(\{g\}\{1\}\{f\}\{w-1\} ;\left\{g+\Delta^{(1)}(i)\right\}\{w\}\right)
\end{gathered}
$$

where the tableau $f^{(N-w)}$ is shown in white in figure 1 . The square added to row $b_{i}=i$ to make $f^{(\mathrm{N}-w+1)}$ is shown with double hatching and the remaining squares added to rows $b_{1} \ldots b_{i-1} b_{i+1} \ldots b_{w}$ with single hatching. The axial distance $\tau_{b_{i} b_{i}}$ is equal to the number of steps to go from the square $b_{j}$ to the square $b_{i}$, the number of arrows in figure 1 , counted positive if to the left and down and negative if to the right and up. Note that

$$
\begin{aligned}
& \tau_{a b}=-\tau_{b a} \\
& \tau_{a b}+\tau_{b c}=\tau_{a c} .
\end{aligned}
$$

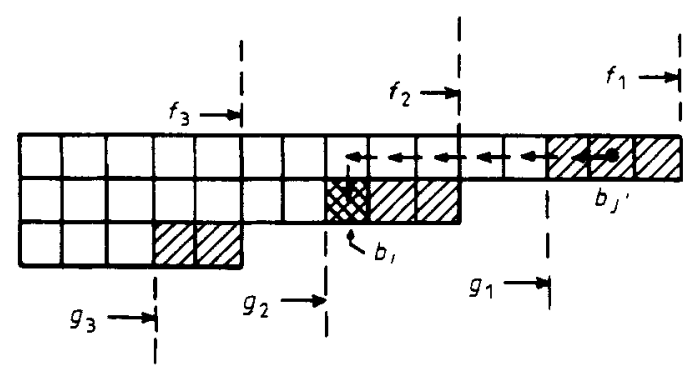

Figure 1. Definition of the axial distance $\tau_{h, h,}$. 
Thus for the product of all $b$, in row $k$ and $k$ smaller than the row of $b_{i}=i$

$$
\prod_{j}\left(1+1 / \tau_{b, b_{1}}\right)=\frac{f_{k}-g_{i}+i-k}{g_{k}-g_{i}+i-k} .
$$

Note that for $k$ greater than $i$, the expression, with a change of sign in both the numerator and the denominator, still holds. For $k=i$, the product (A8) reduces to $\left(f_{i}-g_{i}\right)$. Thus

$$
U\left(\{g\}\{1\}\{f\}\{w-1\} ;\left\{g+\Delta^{(1)}(i)\right\}\{w\}\right)=\left(\frac{1}{w} \frac{\Pi_{k=1}^{n}\left(f_{k}-g_{i}-k+i\right)}{\prod_{k=1, k \neq i}^{n}\left(g_{k}-g_{i}-k+i\right)}\right)^{1 / 2} .
$$

Similarly, using $(\mathrm{A} 3 b)$, we find

$$
U\left(\{g\}\{w-1\}\{f\}\{1\} ;\left\{f-\Delta^{(1)}(i)\right\}\{w\}\right)=\left(\frac{1}{w} \frac{\Pi_{k=1}^{n}\left(f_{i}-g_{k}+k-i\right)}{\prod_{k=1, k \neq i}^{n}\left(f_{i}-f_{k}+k-i\right)}\right)^{1 / 2} .
$$

In the antisymmetric case, the wavefunction is given in a $S_{k} \times$ $\left(S_{N-k} \supset S_{N-k-1} \supset \ldots \supset S_{2} \supset S_{1}\right)$ basis:

$$
\begin{aligned}
& \left.\left.\right|_{m} ^{\{f\}} ;\left\{a_{1} a_{2} \ldots a_{k}\right\}_{\left\{1_{k}\right\}} y_{N-k} y_{N-k-1} \ldots y_{1}\right\rangle \\
& =\sum_{i=1}^{k}\left(\prod_{\substack{j=1 \\
j \neq i}}^{k} S(j-i)\right)\left(\frac{1}{k} \sum_{\substack{j=1 \\
j \neq i}}^{k}\left(1-1 / \tau_{a, a_{1}}\right)\right)^{1 / 2} \\
& \left.\times\left.\right|_{m} ^{\{f\}} ;\left\{a_{1} a_{2} \ldots a_{i-1} a_{i+1} \ldots a_{k}\right\}_{\left\{1_{k-1}\right\}} a_{i} y_{N-k} y_{N-k-1} \ldots y_{1}\right\rangle \\
& =\sum_{i=1}^{k}\left(\prod_{\substack{j=1 \\
j \neq i}}^{k} S(i-j)\right)\left(\frac{1}{k} \prod_{\substack{j=1 \\
j \neq i}}^{k}\left(1-1 / \tau_{a_{1} a_{j}}\right)\right)^{1 / 2} \\
& \left.\times\left.\right|_{m} ^{\{f\}} ; a_{i}\left\{a_{1} a_{2} \ldots a_{i-1} a_{i+1} \ldots a_{k}\right\}_{\left\{1_{k-1}\right.} y_{N-k} y_{N-k-1} \ldots y_{1}\right\rangle
\end{aligned}
$$

where the phase of the initial $S_{k} \times S_{N-k}$ state is fixed through the convention

$$
a_{1}<a_{2}<\ldots<a_{k}
$$

and

$$
S(i-j)= \begin{cases}+1 & i \geqslant j \\ -1 & i<j .\end{cases}
$$

From (A11a), using the analogues of (A4) and (A5), we find

$$
\begin{aligned}
U\left(\{g\}\{1\}\{f\}\left\{1_{k-1}\right\} ;\left\{g+\Delta^{(1)}\left(a_{i}\right)\right\}\left\{1_{k}\right\}\right. \\
=\left(\prod_{\substack{j=1 \\
j \neq i}}^{k} S(j-i)\right)\left(\frac{1}{k} \prod_{\substack{j=1 \\
j \neq i}}^{k} \frac{\left(f_{a_{i}}-f_{a_{j}}+a_{j}-a_{i}+1\right)}{\left(f_{a_{i}}-f_{a_{j}}+a_{j}-a_{i}\right)}\right)^{1 / 2}
\end{aligned}
$$

and, from $(A 11 b)$,

$$
\begin{aligned}
U\left(\{g\}\left\{1_{k-1}\right\}\{f\}\{1\} ;\left\{f-\Delta^{(1)}\left(a_{i}\right)\right\}\left\{1_{k}\right\}\right) \\
=\left(\prod_{\substack{j=1 \\
j \neq i}}^{k} S(i-j)\right)\left(\frac{1}{k} \prod_{\substack{j=1 \\
j \neq i}}^{k} \frac{\left(f_{a_{j}}-f_{a_{i}}+a_{i}-a_{j}+1\right)}{\left(f_{a_{j}}-f_{a_{1}}+a_{i}-a_{j}\right)}\right)^{1 / 2} .
\end{aligned}
$$


For the third class of Racah coefficients $U\left(\{w\}\left\{f_{2}\right\}\{f\}\left\{1_{k}\right\} ;\left\{f_{12}\right\}\left\{f_{23}\right\}\right)$ where $\{f\}$ is an arbitrary $U(n)$ unirrep and where $\{w\}$ and $\left\{1_{k}\right\}$ are respectively symmetrical and antisymmetrical unirreps, we consider for the bra side

$$
\begin{aligned}
& \left.\left\langle{ }_{m}^{\{f}\right\} ; b_{1}^{\prime} \ldots b_{w}^{\prime}\right\}_{\{w\}}\left\{a_{1}^{\prime} \ldots a_{k}^{\prime}\right\}_{\left\{1_{k} k\right.} y_{N-w-k} \ldots y_{1}|\mathbf{r}\rangle \\
& =\left[\left[\left[\psi^{*}\left(\boldsymbol{r}_{1}\right)^{\{1\}} \times \ldots \times \psi^{*}\left(\boldsymbol{r}_{N-w-k}\right)^{\{1\}}\right]^{\left\{f_{2}\right\}} \psi^{*}\left(\boldsymbol{r}_{N-w-k+1}, \ldots, \boldsymbol{r}_{N-w^{*}}\right)^{\left\{1_{k}\right\}}\right]^{\left\{f_{23}\right\}}\right. \\
& \left.\times \psi^{*}\left(\boldsymbol{r}_{N-w+1}, \ldots, \boldsymbol{r}_{N}\right)^{\{w\}}\right]_{m}^{\{f\}} \\
& =\sum_{\left\{f_{13}\right\} \rho_{2,13}} U\left(\left\{f_{2}\right\}\left\{1_{k}\right\}\{f\}\{w\} ;\left\{f_{23}\right\}_{--}\left\{f_{13}\right\}_{-} \rho_{2,13}\right) \\
& \times\left[\left[\psi^{*}\left(\boldsymbol{r}_{1}\right)^{\{1\}} \times \ldots \times \psi^{*}\left(\boldsymbol{r}_{N-w-k}\right)^{\{1\}}\right]^{\left\{f_{2}\right\}}\right. \\
& \left.\times\left[\psi^{*}\left(\boldsymbol{r}_{N-\boldsymbol{w}-k+1}, \ldots, \boldsymbol{r}_{N-\boldsymbol{w}}\right)^{\left\{1_{k}\right\}} \psi^{*}\left(\boldsymbol{r}_{N-w+1}, \ldots, \boldsymbol{r}_{N}\right)^{\{\boldsymbol{w}\}}\right]^{\left\{f_{13}\right\}}\right]_{m}^{\{f\} \rho_{2,13}}
\end{aligned}
$$

and for the ket side

$$
\begin{aligned}
& \left\langle\left.\boldsymbol{r}\right|_{m} ^{\{f\}} ;\left\{a_{1} \ldots a_{k}\right\}_{\left\{1_{k}\right\}}\left\{b_{1} \ldots b_{w}\right\}_{\{w\}} y_{N-w-k} \ldots y_{1}\right\rangle \\
& =\left[\left[\left[\psi\left(\boldsymbol{r}_{1}\right)^{\{1\}} \times \ldots \times \psi\left(r_{N-\boldsymbol{w}-k}\right)^{\{1\}}\right]^{\left\{f_{2}\right\}} \psi\left(\boldsymbol{r}_{N-\boldsymbol{w}-k+1}, \ldots, \boldsymbol{r}_{N-k}\right)^{\{\boldsymbol{w}\}}\right]^{\left\{f_{12}\right\}}\right. \\
& \left.\times \psi\left(\boldsymbol{r}_{N-k+1}, \ldots, \boldsymbol{r}_{N}\right)^{\left\{1_{k}\right\}}\right]_{m}^{\{f\}} \\
& =\sum_{\left\{f_{13}\right\} \rho_{2,13}} U\left(\left\{f_{2}\right\}\{w\}\{f\}\left\{1_{k}\right\} ;\left\{f_{12}\right\}_{--}\left\{f_{13}\right\}_{-} \rho_{2,13}\right) \\
& \times\left[\left[\psi\left(\boldsymbol{r}_{1}\right)^{\{1\}} \times \ldots \times \psi\left(\boldsymbol{r}_{\mathbf{N}-\boldsymbol{w}-k}\right)^{\{1\}}\right]^{\left\{f_{2}\right\}}\right. \\
& \left.\times\left[\psi\left(\boldsymbol{r}_{N-w-k+1}, \ldots, \boldsymbol{r}_{N-k}\right)^{\{w\}} \psi\left(\boldsymbol{r}_{N-k+1}, \ldots, \boldsymbol{r}_{N}\right)^{\left\{1_{k}\right\}}\right]^{\left\{f_{13} i\right.}\right]_{m}^{\{f\} \rho_{2,13}} \\
& =\sum_{\left\{f_{13}\right\} \rho_{2,13}}(-1)^{\phi\left(\left\{1_{k}\right\}\right)+\phi(\{w\})-\phi\left(\left\{f_{13}\right\}\right)} U\left(\left\{f_{2}\right\}\{w\}\{f\}\left\{1_{k}\right\} ;\left\{f_{12}\right\}_{--}\left\{f_{13}\right\}_{-} \rho_{2,13}\right) \\
& \times\left[\left[\psi\left(\boldsymbol{r}_{1}\right)^{\{1\}} \times \ldots \times \psi\left(\boldsymbol{r}_{N-w-k}\right)^{\{1\}}\right]^{\left\{f_{2}\right\}}\right. \\
& \left.\times\left[\psi\left(\boldsymbol{r}_{N-k+1}, \ldots, \boldsymbol{r}_{N}\right)^{\left\{1_{k}\right\}} \psi\left(\boldsymbol{r}_{N-\boldsymbol{w}-k+1}, \ldots, \boldsymbol{r}_{N-k}\right)^{\{\boldsymbol{w}\}}\right]^{\left\{f_{13}\right\}}\right]_{m}^{\{f\} \rho_{2,13}}
\end{aligned}
$$

(see the appendix of $\mathrm{I}$ for the last rearrangement phase). The multiplicity label $\rho_{a b}$ in

$$
U\left(\left\{f_{1}\right\}\left\{f_{2}\right\}\{f\}\left\{f_{3}\right\} ;\left\{f_{12}\right\} \rho_{12} \rho_{12,3}\left\{f_{23}\right\} \rho_{23} \rho_{1,23}\right)
$$

indicates that in general there is a multiplicity to be considered for the coupling $\{a\} \otimes\{b\}$ while its absence (denoted _ ) indicates that the coupling is multiplicity free.

To obtain the state (A16) in a form in which it can be evaluated through an overlap norm with state (A15), let it be acted upon by the product $\mathbf{P}$ of permutation operators which rearranges the particle numbers in the last two functions. Assuming $k \leqslant w$ (if otherwise, we would reverse the role of the bra (A15) and the ket (A16))

$\mathbf{P}=\mathscr{P}_{k} \ldots \mathscr{P}_{2} \mathscr{P}_{1} P_{N(N-w)} P_{(N-1)(N-w-1)} \ldots P_{(N-k+2)(N-w-k+2)} P_{(N-k+1)(N-w-k+1)}$

where $\mathscr{P}_{s}$ are cyclic interchanges of the $(w-k)$ particles labelled $N-s+1, N-k$, $N-k-1, \ldots, N-w+1$. Thus

$$
\begin{aligned}
\mathbf{P}\left[\psi\left(\boldsymbol{r}_{N-k+1}, \ldots, \boldsymbol{r}_{N}\right)^{\left\{\mathbf{1}_{k}\right\}} \psi\left(\boldsymbol{r}_{N-w-k+1}, \ldots, \boldsymbol{r}_{N-k}\right)^{\{w\}}\right]_{m_{13}\left\{f_{13}\right\}} & =\left[\psi\left(\boldsymbol{r}_{N-w-k+1}, \ldots, \boldsymbol{r}_{N-w}\right)^{\left\{1_{k}\right\}} \psi\left(\boldsymbol{r}_{N-w+1}, \ldots, \boldsymbol{r}_{N}\right)^{\{\boldsymbol{w}\}}\right]_{m_{13}\left\{f_{13}\right\}}
\end{aligned}
$$


and (A15) and (A16) give

$$
\begin{aligned}
& \left\langle{ }_{m}^{\{f\}} ;\left\{b_{1}^{\prime} \ldots b_{w}^{\prime}\right\}_{\{w\}}\left\{a_{1}^{\prime} \ldots a_{k}^{\prime}\right\}_{\left\{1_{k}\right\}} y_{N-w-k} \ldots y_{1}|\mathbf{P}|_{m}^{\{f\}} ;\left\{a_{1} \ldots a_{k}\right\}_{\left\{1_{k}\right\}}\right. \\
& \left.\times\left\{b_{1} \ldots b_{w}\right\}_{\{w \mid} y_{N-w-k} \ldots y_{1}\right\rangle \\
& =\sum_{\left\{f_{1}\right\} \rho_{2,13}}(-1)^{\phi\left(\left\{1_{k}\right\}\right)+\phi\{\{w\})-\phi\left\{\left\{f_{13}\right\}\right)} \\
& \times U\left(\left\{f_{2}\right\}\left\{1_{k}\right\}\{f\}\{w\} ;\left\{f_{23}\right\}_{--}\left\{f_{13}\right\}_{-} \rho_{2,13}\right) \\
& \times U\left(\left\{f_{2}\right\}\{w\}\{f\}\left\{1_{k}\right\} ;\left\{f_{12}\right\}_{--}\left\{f_{13}\right\}_{-} \rho_{2,13}\right) \\
& =(-1)^{\phi\left(\left\{f_{2}\right\}\right)+\phi(\{w\})-\phi\left(\left\{f_{12}\right\}\right)}(-1)^{\phi(\{f\})-\phi(\{w\})-\phi\left(\left\{f_{23}\right\}\right)} \\
& \times U\left(\{w\}\left\{f_{2}\right\}\{f\}\left\{1_{k}\right\} ;\left\{f_{12}\right\}\left\{f_{23}\right\}\right)
\end{aligned}
$$

where the last step has made use of a standard composition of $\mathrm{U}(n)$ recoupling transformations (see (6) and figure 1 of Hecht (1975)).

From (A15), (A16), (A18) and (A19)

$$
\begin{aligned}
\left\langle{ }_{m}^{\{f\}} ;\left\{b_{1}^{\prime} \ldots b_{w}^{\prime}\right\}_{\{w\}}\right. & \left\{a_{1}^{\prime} \ldots a_{k}^{\prime}\right\}_{\left\{1_{k}\right\}} y_{N-w-k} \ldots y_{1}|\mathbf{P}|_{m}^{\{f\}} ;\left\{a_{1} \ldots a_{k}\right\}_{\left\{1_{k}\right\}} \\
& \left.\times\left\{b_{1} \ldots b_{w}\right\}_{\{w\}} y_{N-w-k} \ldots y_{1}\right\rangle \\
= & (-1)^{\phi\left(\left\{f_{2}\right\}\right)+\phi(\{w\})-\phi\left(\left\{f_{12}\right\}\right)}(-1)^{\phi(\{f\})-\phi(\{w\})-\phi\left(\left\{f_{23}\right\}\right)} \\
& \times U\left(\{w\}\left\{f_{2}\right\}\{f\}\left\{1_{k}\right\} ;\left\{f_{12}\right\}\left\{f_{23}\right\}\right)
\end{aligned}
$$

which will yield the desired quantity. Since a $\mathscr{P}_{s}$ in (A17) acting to the left on a totally symmetric state yields +1 , it remains to evaluate

$$
\prod_{t=1}^{k} P_{N-t+1, N-t-w+1}\left|\{f\} ;\left\{a_{1} \ldots a_{k}\right\}_{\left\{1_{k}\right\}}\left\{b_{1} \ldots b_{w}\right\}_{\{w\}} y_{N-w-k} \ldots y_{1}\right\rangle .
$$

This can be evaluated with the use of (A11) and

$$
\begin{aligned}
&\left.\left.P_{N-m, N-m-w}\right|_{m} ^{\{f\}} ; y_{N} \ldots y_{N-m+1} a\left\{b_{1} \ldots b_{w}\right\}_{\{w\}} y_{N-m-w-1} \ldots y_{1}\right\rangle \\
&=\left.\left.\left(\prod_{j=1}^{w}\left(1-1 / \tau_{a b_{1}}^{2}\right)\right)^{1 / 2}\right|_{m} ^{\{f\}} ; y_{N} \ldots y_{N-m+1}\left\{b_{1} \ldots b_{w}\right\}_{\{w\}} a y_{N-m-w-1} \ldots y_{1}\right\rangle \\
&+\sum_{s=1}^{w} \frac{1}{\tau_{a b_{1}}}\left(\prod_{\substack{j=1 \\
j \neq s}}^{w}\left(1+1 / \tau_{a b_{1}}\right)\left(1+1 / \tau_{b_{i}, b_{1}}\right)\right)^{1 / 2} \\
&\left.\times\left.\right|_{m} ^{\{f\}} ; y_{N} \ldots y_{N-m+1}\left\{a b_{1} \ldots b_{s-1} b_{s+1} \ldots b_{w}\right\}_{\{w\}} b_{s} y_{N-m-x^{*}-1} \ldots y_{1}\right\rangle
\end{aligned}
$$

(see equation (23) of Hecht (1975)).

In the most general case, illustrated by figure $2(a)$, with

$$
\left\{a_{1} a_{2} \ldots a_{k}\right\}=\left\{i_{1} i_{2} \ldots i_{k}\right\} \quad i_{1}<i_{2}<\ldots<i_{k}
$$

and

$$
\left\{a_{1}^{\prime} a_{2}^{\prime} \ldots a_{k}^{\prime}\right\}=\left\{j_{1} j_{2} \ldots j_{k}\right\} \quad j_{1}<j_{2}<\ldots<j_{k}
$$

and with all $a_{s}^{\prime}$ different from all $a_{s}$, the set of $k i$ and $k j$ never occupy a common square in the Young tableau. Equation (A20) leads to the evaluation of the Racah coefficient

$$
U\left(\{w\}\left\{m-\Delta^{(k)}(j)\right\}\{f\}\left\{1_{k}\right\} ;\left\{f-\Delta^{(k)}(\boldsymbol{i})\right\}\{m\}\right)
$$



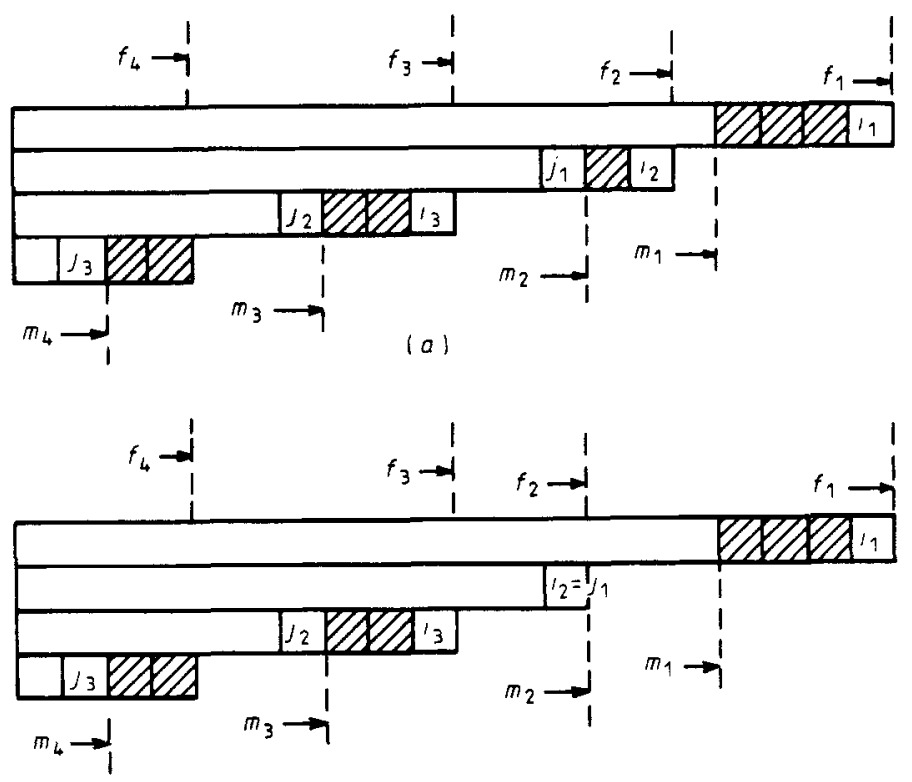

(b)

Figure 2. Young tableaux corresponding to the Racah coefficient (A20). (a) General case; (b) special case.

where $\left\{f-\Delta^{(k)}(i)\right\}$ designates a tableau with one square removed from rows $i_{1}, \ldots, i_{k}$; similarly for $\left\{m-\Delta^{(k)}(j)\right\}$, as illustrated by figure $2(a)$.

In this case for each $i_{s}$, there is at least one member of the set $\left\{b_{1} \ldots b_{w}\right\}$ for which $\tau_{i, b}=1$ so that only the second term of (A21) gives a non-zero contribution. Repeated application of (A21) then yields

$$
\begin{aligned}
\prod_{t=1}^{k} P_{N-t+1, N-t-w+1}\left|\left\{i_{1} i_{2} \ldots i_{k}\right\}_{\left\{1_{k}\right\}}\left\{j_{1} j_{2} \ldots j_{k} c_{1} \ldots c_{w-k}\right\}_{\{w\}} y_{N-w-k} \ldots y_{1}\right\rangle \\
=F_{k}(i, j)\left|\left\{i_{1} i_{2} \ldots i_{k} c_{1} \ldots c_{w-k}\right\}_{\{w\}}\left\{j_{1} j_{2} \ldots j_{k}\right\}_{\left\{1_{k}\right\}} y_{N-w-k} \ldots y_{1}\right\rangle \\
\quad+\text { unwanted terms }
\end{aligned}
$$

where

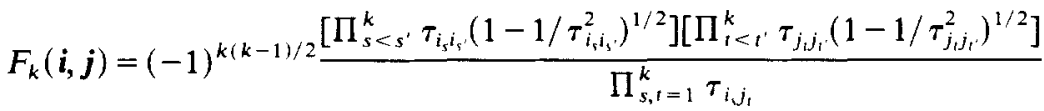

$$
\begin{aligned}
& \times\left(\prod_{t=1}^{k} \prod_{r=1}^{w-k}\left(1+1 / \tau_{c_{r} j_{t}}\right)^{1 / 2}\right)\left(\prod_{s=1}^{k} \prod_{r=1}^{w-k}\left(1+1 / \tau_{i, c_{r}}\right)^{1 / 2}\right) .
\end{aligned}
$$

Note that the squares labelled by the $c_{r}$ are illustrated by hatched squares in figure 2. The various factors in (A22b) have the following values:

$$
\begin{aligned}
& \tau_{i_{s} j_{t}}=S\left(j_{t}-i_{s}\right)\left(f_{i_{s}}-m_{j_{t}}+j_{t}-i_{s}\right) \\
& \tau_{i_{s} i_{s}}\left(1-1 / \tau_{i_{s} i_{r}}^{2}\right)^{1 / 2}=\left[\left(f_{i_{s}}-f_{i_{,}}+i_{s^{\prime}}-i_{s}+1\right)\left(f_{i,}-f_{i_{v^{\prime}}}+i_{s^{\prime}}-i_{s}-1\right)\right]^{1 / 2} \quad s<s^{\prime} \\
& \tau_{j, j_{1}}\left(1-1 / \tau_{j_{t} j_{t^{\prime}}}^{2}\right)^{1 / 2}=\left[\left(m_{j_{t}}-m_{j_{,}}+j_{t^{\prime}}-j_{t}+1\right)\left(m_{j_{1}}-m_{j_{t^{\prime}}}+j_{t^{\prime}}-j_{t}-1\right)\right]^{1 / 2} \quad t<t^{\prime}
\end{aligned}
$$




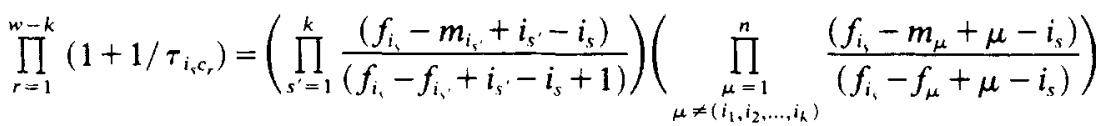

$$
\begin{aligned}
& \prod_{r=1}^{w-k}\left(1+1 / \tau_{c_{r_{j}}}\right)=\left(\prod_{s=1}^{k} \frac{\left(f_{i_{s}}-m_{j_{t}}+j_{t}-i_{s}\right)}{\left(m_{i_{s}}-m_{j_{t}}+j_{t}-i_{s}+1\right)}\right)\left(\prod_{\substack{\mu=1 \\
\mu \neq\left(j_{1}, j_{2}, \ldots, j_{k}\right)}}^{n} \frac{\left(f_{\mu}-m_{j_{t}}+j_{t}-\mu+1\right)}{\left(m_{\mu}-m_{j_{t}}+j_{t}-\mu+1\right)}\right)
\end{aligned}
$$

leading to

$$
\begin{aligned}
& F_{k}(i, j)=(-1)^{k(k-1) / 2}\left(\prod_{s, t=1}^{k} S\left(j_{t}-i_{s}\right)\right) \\
& \times \prod_{s=1}^{k}\left(\prod_{\substack{\mu=1 \\
\mu \neq\left(i_{1}, i_{2}, \ldots, i_{k}\right)}}^{n} \frac{\left(f_{\mu}-m_{j_{s}}+j_{s}-\mu+1\right)}{\left(f_{i_{s}}-f_{\mu}+\mu-i_{s}\right)}\right. \\
& \left.\times \prod_{\substack{\nu=1 \\
\nu \neq\left(j_{1}, j_{2}, \ldots, j_{k}\right)}}^{n} \frac{\left(f_{i_{s}}-m_{\nu}+\nu-i_{s}\right)}{\left(m_{\nu}-m_{j_{s}}+j_{s}-\nu+1\right)}\right)^{1 / 2} .
\end{aligned}
$$

In the special case, illustrated in figure $2(b)$, when one of the $i_{s}$ (e.g. $i_{q}$ ) occupies the same square as one of the $j_{t}$ (e.g. $j_{v}$ ), action with $P_{N-k+1, N-k-w+1}$ requires both terms of (A21) but action with all subsequent operators within $\mathbf{P}$ requires only the second term of $(\mathrm{A} 21)$. In the special case when $i_{q}=j_{v}$ (i.e. $i_{q}$ occupies the same square as $j_{v}$ in the Young tableau), the matrix element of $\mathbf{P}$ is

$$
\begin{aligned}
\left\langle\left\{ i_{1} \ldots i_{q-1} i_{q+1}\right.\right. & \left.\ldots i_{k} c_{1} \ldots c_{w-k+1}\right\}_{\{w\}}\left\{j_{1} \ldots j_{v} \ldots j_{k}\right\}_{\left\{1_{k}\right.} y_{N-w-k} \ldots y_{1} \mid \\
& \times \mathbf{P}\left\{\left\{i_{1} \ldots i_{q} \ldots i_{k}\right\}_{\left\{1_{k}\right\}}\left\{j_{1} \ldots j_{v-1} j_{v+1} \ldots j_{k} c_{1} \ldots c_{w-k+1}\right\}_{\{w\}} y_{N-w-k} \ldots y_{1}\right\rangle \\
& =F_{k}(i, j)
\end{aligned}
$$

where $F_{k}(i, j)$ is identical with that given in (A24). Iterating this process, we can see that the same result holds when two or more of the $i$ occupy the same squares as two of the $j$ up to the special case when all $k i$ occupy the same squares as the $k j$.

Finally, using the phase factor relations

$$
\begin{aligned}
& \phi(\{f\})-\phi\left(\left\{f-\Delta^{(k)}(i)\right\}=\frac{1}{2} \sum_{a=1}^{n}(n+1-2 a) f_{a}-\frac{1}{2} \sum_{a=1}^{n}(n+1-2 a)\left(f_{a}+\Delta_{a}^{(k)}(i)\right)\right. \\
& =+\frac{1}{2} k(n+1)-\sum_{s=1}^{k} i_{s} \\
& \phi\left(\left\{m-\Delta^{(k)}(j)\right\}\right)-\phi(\{m\})=-\frac{1}{2} k(n+1)+\sum_{s=1}^{k} j_{s}
\end{aligned}
$$

for the phase in (A19), the general result for the $\mathrm{U}(n)$ Racah coefficient of type (3) follows:

$$
\begin{aligned}
& U\left(\{w\}\left\{m-\Delta^{(k)}(j)\right\}\{f\}\left\{1_{k}\right\} ;\left\{f-\Delta^{(k)}(i)\right\}\{m\}\right) \\
&=(-1)^{k(k-1) / 2+\Sigma_{s=1}^{k}\left(j_{s}-i_{s}\right)}\left(\prod_{s, t=1}^{k} S\left(j_{1}-i_{s}\right)\right) \\
& \times \prod_{s=1}^{k}\left(\prod_{\substack{\mu=1 \\
\mu \neq\left(i_{1}, i_{2}, \ldots, i_{k}\right)}}^{n} \frac{\left(f_{\mu}-m_{j_{s}}+j_{s}-\mu+1\right)}{\left(f_{\mu}-f_{i_{s}}+i_{s}-\mu\right)}\right. \\
&\left.\times \prod_{\substack{\nu=1 \\
\nu \neq\left(j_{1}, j_{2}, \ldots, j_{k}\right)}}^{n} \frac{\left(m_{\nu}-f_{i_{s}}+i_{s}-\nu\right)}{\left(m_{\nu}-m_{j_{s}}+j_{s}-\nu+1\right)}\right)^{1 / 2} .
\end{aligned}
$$


With $\{f\}=\left\{m^{\circ}\right\}$, for a $\mathrm{U}(n-1)$ Racah coefficient as in $\$ 3$ and in terms of the hook (see (2.7)), we obtain

$$
\begin{aligned}
& U\left(\{w\}\left\{m-\Delta^{(k)}(j)\right\}\left\{m^{o}\right\}\left\{1_{k}\right\} ;\left\{m^{o}-\Delta^{(k)}(i)\right\}\{m\}\right) \\
&=(-1)^{k(k-1) / 2+\Sigma_{,=1}^{k}\left(j_{s}-i_{s}\right)}\left(\prod_{s, t=1}^{k} S\left(j_{t}-i_{s}\right)\right) \\
& \times \prod_{s=1}^{k}\left(\prod_{\substack{\mu=1 \\
\mu \neq\left(i_{1}, i_{2}, \ldots, i_{k}\right)}}^{n-1} \frac{\left(p_{\mu n}-p_{j_{s}, n-1}\right)}{\left(p_{\mu n}-p_{i_{,}, n}\right)} \prod_{\substack{\nu=1 \\
\nu \neq\left(j_{1}, j_{2}, \ldots, j_{k}\right)}}^{n-1} \frac{\left(p_{\nu, n-1}-p_{i_{s},}+1\right)}{\left(p_{\nu, n-1}-p_{j, n-1}+1\right)}\right)^{1 / 2} .
\end{aligned}
$$

\section{References}

Baird G E and Biedenharn L C 1963 J. Math. Phys. 41443

Bargmann V 1962 Rev. Mod. Phys. 34829

Biedenharn L C, Giovanninni A and Louck J D 1967 J. Math. Phys. 8691

Biedenharn L C, Lohe M A and Louck J D 1985 J. Math. Phys. 261458

Biedenharn L C and Louck J D 1968 Commun. Math. Phys. 889 1972 J. Math. Phys. 131985

Biedenharn L C, Louck J D, Chaćon E and Ciftan M 1972 J. Math. Phys. 131957

Hecht K T 1975 Commun. Math. Phys. 41135

Hecht K T, Le Blanc R and Rowe D J 1987a J. Phys. A: Math. Gen. 20257

1987b J. Phys. A: Math. Gen. 202241

Horie H 1964 J. Phys. Soc. Japan 191783

Kramer P 1967 Z. Phys. 205181

Le Blanc R 1987 J. Phys. A: Math. Gen. 205015

Le Blanc R and Hecht K T 1986 Preprint University of Michigan NP86102

Le Blanc R and Rowe D J 1985a J. Phys. A: Math. Gen. 181891

_ 1985b J. Phys. A: Math. Gen. 181905

- 1986a J. Phys. A: Maih. Gen. 191093

1986b J. Phys. A: Math. Gen. 192913

Louck J D 1970 Am. J. Phys. 383

Louck J D and Biedenharn L C 1973 J. Math. Phys. 141336

Moshinsky M 1963 J. Math. Phys. 41128 